\title{
Connections between Anterior Inferotemporal Cortex and Superior Temporal Sulcus Regions in the Macaque Monkey
}

\author{
K. S. Saleem, ${ }^{1,2}$ W. Suzuki, ${ }^{1,3}$ K. Tanaka, ${ }^{1,4}$ and T. Hashikawa ${ }^{1}$ \\ ${ }^{1}$ Riken Brain Science Institute, Wako, Saitama 351-0198, Japan, 2Brain Science and Life Technology Research \\ Foundation, Wako, Saitama 351-0198, Japan, ${ }^{3}$ Graduate School of Engineering Sciences, Osaka University, Toyonaka, \\ Osaka 560-0043, Japan, and ${ }^{4}$ Core Research for Evolutional Science and Technology, Japan Science and Technology \\ Corporation, Wako, Saitama 351-0198, Japan
}

\begin{abstract}
We examined the connections between the anterior inferotemporal cortex and the superior temporal sulcus (STS) in the macaque monkey by injecting Phaseolus vulgaris leucoagglutinin (PHA-L) or wheat germ agglutinin conjugated to horseradish peroxidase (WGA-HRP) into the dorsoanterior and ventroanterior subdivisions of TE (TEad and TEav, respectively) and observing the labeled terminals and cell bodies in STS. We found a clear dichotomy in the connections of the rostral part of STS: the injections into TEad resulted in a dense distribution of labeled terminals and cell bodies in the upper bank of rostral STS, whereas labeling was confined to the lower bank and fundus of rostral STS after injections into TEav. The distribution of labeling in the rostral STS was discontinuous from the distribution of labeling surrounding the injection sites: the lower bank of the rostral STS was spared from labeling in the TEad injection cases, and TEad had only sparse distribution in the
\end{abstract}

TEav injection cases. These results revise the classical view that the lower bank of rostral STS is connected with TE, whereas the upper bank of rostral STS is connected with the parietal, prefrontal, and superior temporal regions (Seltzer and Pandya, 1978, 1991, 1994). The upper bank of the rostral STS is called the superior temporal polysensory area (STP), because it was previously found that neurons there respond to auditory, somatosensory, and visual stimuli. The present results thus suggest that the polymodal representation in STP interacts more with information processing in TEad than TEav. It is also suggested that the information processing in the ventral bank of the rostral STS is distinct from that in TEad, and the former more directly interacts with TEav than TEad.

Key words: inferotemporal cortex; area TE; superior temporal sulcus; polysensory area; PHA-L; WGA-HRP; laminar organization; macaque monkey
Inferotemporal area TE of the macaque monkey is an extrastriate visual cortical area that represents the final purely visual stage in the occipitotemporal pathway (Gross, 1994). TE is thought to be important for object vision, i.e., the discrimination and recognition of visual images of objects. Cells in TE selectively respond to complex features of object images, and cells with similar selectivity are clustered in columnar regions in TE (for review, see Tanaka, 1993, 1997). Area TE projects to numerous brain sites, including the perirhinal cortex, prefrontal cortex, amygdala, striatum, and superior temporal sulcus (Van Hoesen and Pandya, 1975; Seltzer and Pandya, 1978; Turner et al., 1980; Shiwa, 1987; Saint-Cyr et al., 1990; Yukie et al., 1990; Webster et al., 1991, 1994; Suzuki and Amaral, 1994; Saleem and Tanaka, 1996; Cheng et al., 1997).

It has recently been found that the dorsal and ventral subregions of anterior TE (TEad and TEav, respectively) differentially project to the perirhinal and entorhinal cortices (Saleem and Tanaka, 1996), the striatum and amygdala (Yukie et al., 1990; Cheng et al., 1997), the hippocampal formation (Yukie et al., 1990; Saleem and Hashikawa, 1998), and the prefrontal cortex (Saleem et al., 1995). There are also suggestions that TEad and TEav receive afferent inputs from the occipital cortex through

Received Feb. 23, 2000; revised April 10, 2000; accepted April 11, 2000.

This work was supported by the Riken Brain Science Institute. We thank A. H. Asiya Begum for surgical and histological assistance.

Correspondence should be addressed to Dr. K. S. Saleem, Laboratory for Neural Architecture, Riken Brain Science Institute, 2-1 Hirosawa, Wako-shi, Saitama 3510198, Japan. E-mail: saleem@postman.riken.go.jp.

Copyright (C) 2000 Society for Neuroscience $0270-6474 / 00 / 205083-19 \$ 15.00 / 0$ separate pathways (Martin-Elkins and Horel, 1992; Yukie et al., 1992). Moreover, behavioral studies (Horel et al., 1987; Horel, 1994a,b) found that cooling limited to the inferior temporal gyrus, including TEav and the perirhinal cortex, produced deficits in performance on delayed matching-to-sample with visual objects, whereas cooling of TEad produced deficits in discrimination of color and fine shapes (see also Buckley et al., 1997).

In the present study we examined connections of TEad and TEav with the superior temporal sulcus (STS). Both connectional and physiological studies have shown that the upper bank and fundus of STS are polymodal, whereas the lower bank of STS is purely visual (Seltzer and Pandya, 1978, 1991; Desimone and Gross, 1979; Bruce et al., 1981; Baylis et al., 1987). Although it has been shown that the lower bank of rostral STS is connected with TE and the upper bank of rostral STS is connected with the parietal, prefrontal, and superior temporal regions (Seltzer and Pandya, 1978, 1991, 1994; Baizer et al., 1991; Barnes and Pandya, 1992), the detailed organization of these connections has not been studied. In this study we made focal injections of the anterograde tracer Phaseolus vulgaris leucoagglutinin (PHA-L) or the bidirectional tracer wheat germ agglutinin conjugated to horseradish peroxidase (WGA-HRP) into TEad or TEav and observed the areal and laminar distribution of labeled terminals and cell bodies in STS. We found a dichotomy in the connections of TEad and TEav with the rostral STS: TEad connects with the upper bank of rostral STS, whereas TEav connects with the lower bank and fundus of rostral STS. We also examined the connections of 


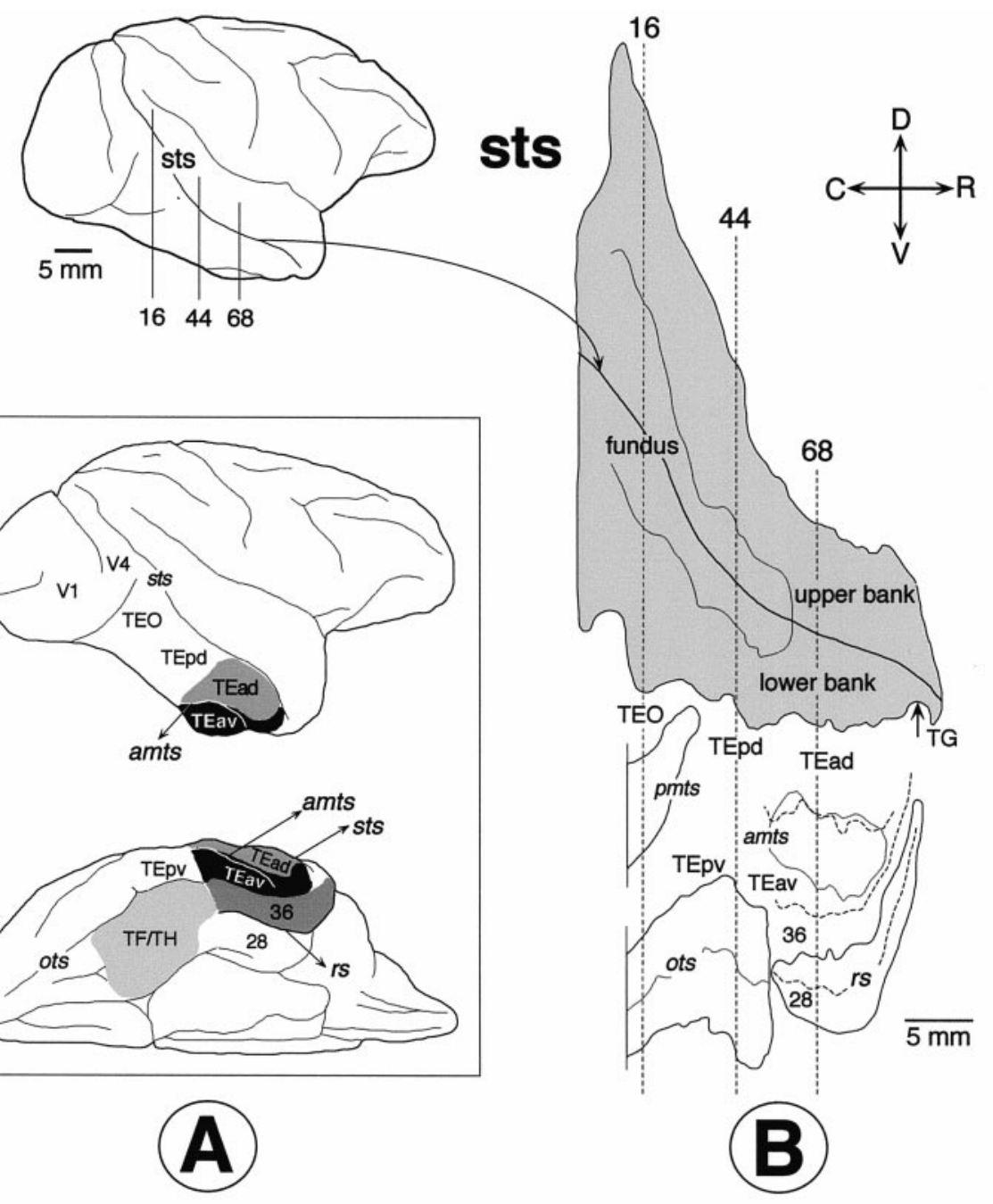

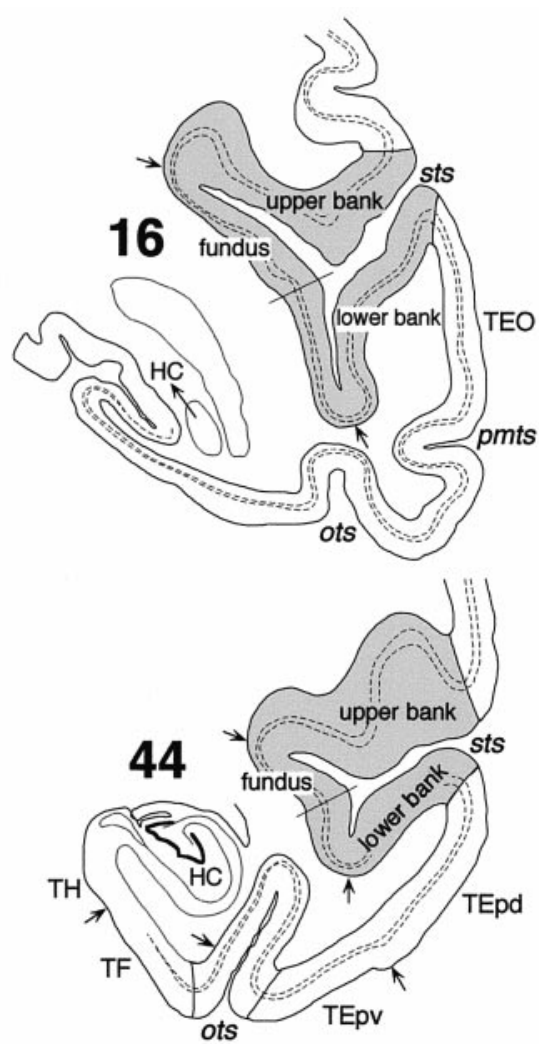

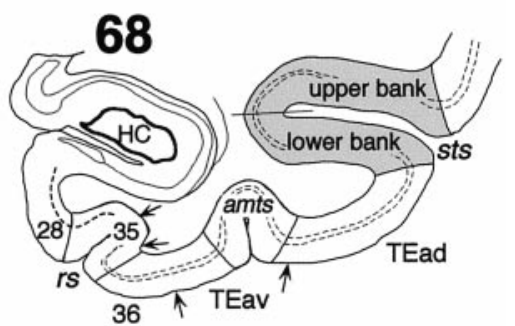

Figure 1. A, Lateral (top) and ventral (bottom) views of the right hemisphere showing the location and extent of the TEad and TEav, perirhinal cortex (areas 35 and 36), and the parahippocampal gyrus $(T F / T H)$. Area 35 is located in the fundus of rhinal sulcus. $B$, Reconstruction of two-dimensional unfolded map, in which the superior temporal sulcus (sts), anterior middle temporal sulcus (amts), posterior middle temporal sulcus (pmts), occipitotemporal sulcus (ots), and rhinal sulcus $(r s)$ are exposed. The solid lines indicate the lips and fundi of the sulci, and the broken lines show the borders between cortical areas. As opposed to conventional unfolding, the coronal sections are represented by vertical straight lines in the unfolded map. Camera lucida drawings of three representative coronal sections are shown on the right (sections 16, 44, and 68). The broken lines in the coronal sections indicate the borders of layer 4. The arrows indicate the borders between different cortical areas. $H C$, Hippocampus; $C$, caudal; $R$, rostral; $D$, dorsal; $V$, ventral.

TEad and TEav with more posterior inferotemporal regions in the ventrolateral surface and STS to examine the differences in their visual afferent pathways.

Some of the present results have been reported previously in abstract form (Saleem et al., 1996).

\section{MATERIALS AND METHODS}

Eleven Japanese monkeys (Macaca fuscata) of both sexes, weighing between 3.3 and $6.9 \mathrm{~kg}$, were used. PHA-L was injected into the TEad in one monkey and the TEav in two other monkeys. WGA-HRP was injected into the TEad in another two monkeys, the TEav in another three monkeys, at the border between the TEav and area 36 of the perirhinal cortex in another monkey, and in area 36 in another monkey. In addition, WGA-HRP was injected into the superior temporal polysensory area (STP) in the upper bank of rostral STS in the last monkey, to confirm the connections of STP with TEad and the absence of STP connections with TEav. The tracers were injected into a single site except in the STP injection case, in which WGA-HRP was injected into two nearby sites to cover a larger part of STP. Some of these cases are shared with other studies conducted in our laboratories (Saleem and Tanaka, 1996; Cheng et al., 1997; Saleem and Hashikawa, 1999).

Surgery and tracer injection. The tracers were injected during aseptic surgery under general anesthesia, as described previously (Saleem and Tanaka, 1996). After pretreatment with atropine sulfate $(0.1 \mathrm{mg} / \mathrm{kg}$, i.m.) and sedation with ketamine hydrochloride $(12 \mathrm{mg} / \mathrm{kg}$, i.m.), each monkey was anesthetized by intraperitoneal injection of sodium pentobarbital (Nembutal, $35 \mathrm{mg} / \mathrm{kg}$ ). Supplemental doses of sodium pentobarbital $(9 \mathrm{mg} / \mathrm{kg}$, i.p.) were given as needed to maintain a surgical level of anesthesia. Tranexamic acid $(25 \mathrm{mg} / \mathrm{kg}$, i.m. $)$ was given to minimize bleeding. The body temperature, heart rate, and respiratory rate were monitored throughout surgery.

STS and the anterior middle temporal sulcus (AMTS) were exposed after craniotomy to use as landmarks for placement of injection site. PHA-L (2.5\%; Vector Laboratories, Burlingame, CA) was injected iontophoretically (Midgard precision current source, Stoelting) according to the procedure recommended by Gerfen and Sawchenko (1984) with some modifications (Saleem et al., 1993). WGA-HRP (5\%; Toyobo, Osaka, Japan) was injected by pressure, according to the method described in Saleem and Tanaka (1996). After the injection was completed, 

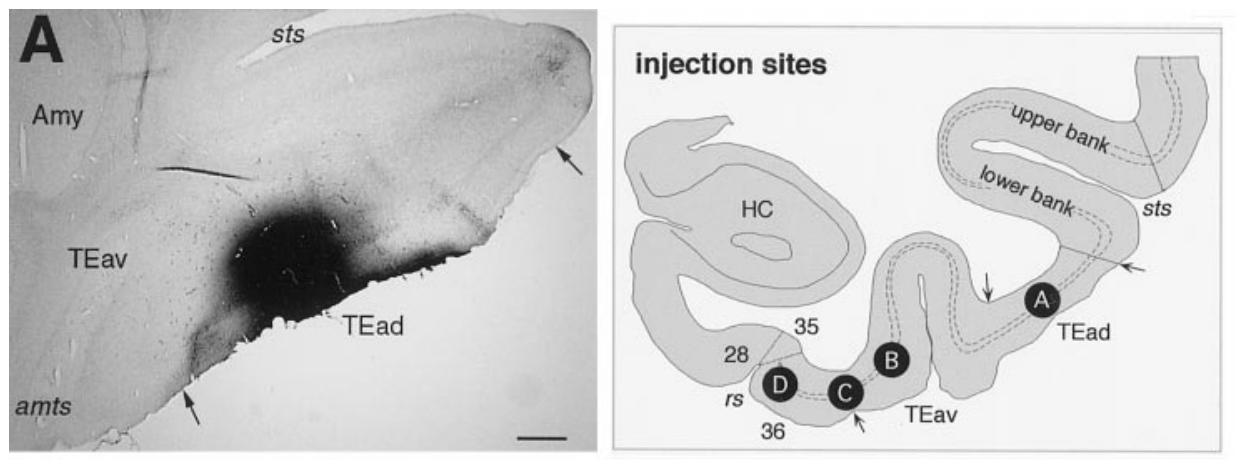
A. TEad: WGA-HRP
B. TEav: WGA-HRP
C. TEav-36 border: WGA-HRP
D. 36: WGA-HRP
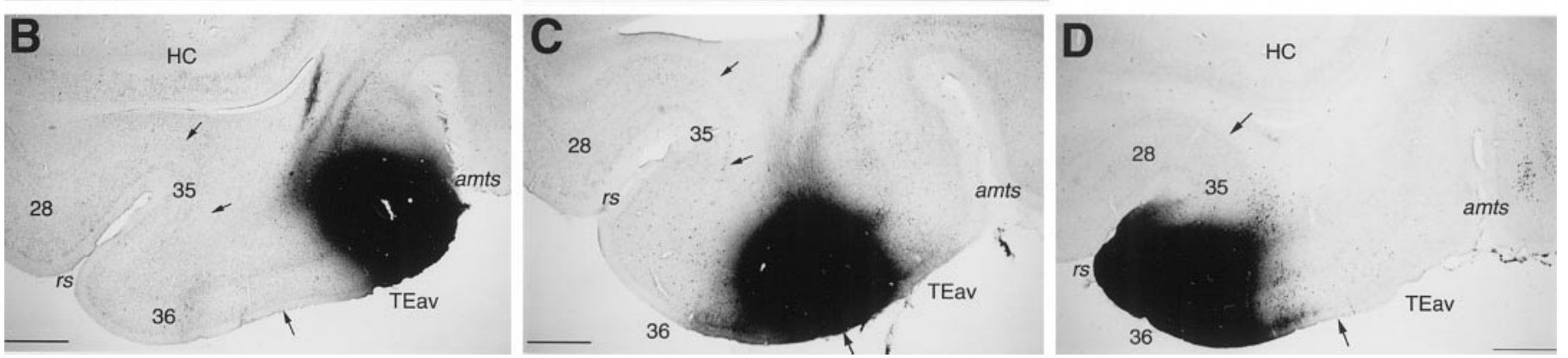

Figure 2. Photomicrographs illustrating the WGA-HRP injection sites in TEad $(A)$, TEav $(B)$, at the border between TEav and area 36 of the perirhinal cortex $(C)$, and in area $36(D)$. The positions of these injection sites are schematically illustrated in the coronal section drawing at the top right. For the PHA-L injection sites, see Saleem and Tanaka (1996), their Figure 6. Scale bars, $1 \mathrm{~mm}$.

the dura was sutured, and the wound was closed. Dexamethasone sodium phosphate $(1 \mathrm{mg} / \mathrm{kg}$, i.m.) was given after the surgery to minimize the cerebral edema. The antibiotic piperacillin sodium $(55 \mathrm{mg} / \mathrm{kg}$, i.m.) and analgesic ketoprofen $(5 \mathrm{mg} / \mathrm{kg}$, i.m.) were injected daily for 4-5 d after the surgery. The experimental protocol had been approved by the Experimental Animal Committee of the RIKEN Institute and had conformed to National Institutes of Health guidelines.

Histological processing. Two days after the WGA-HRP injection and 16-18 d after the PHA-L injections, the monkey received a lethal dose of sodium pentobarbital $(60-80 \mathrm{mg} / \mathrm{kg}$, i.v. $)$ and was perfused transcardially with 11 of $0.9 \%$ warm heparinized saline, followed by 3-4 1 of cold $4 \%$ paraformaldehyde in $0.1 \mathrm{M}$ phosphate buffer, $\mathrm{pH} 7.2-7.4$, then $1-21$ of $10 \%$ sucrose in $0.1 \mathrm{M}$ phosphate buffer, and finally by 11 of $20 \%$ sucrose in $0.1 \mathrm{M}$ phosphate buffer. The flow rate of the fixative solution was adjusted so that the perfusion with paraformaldehyde took 30-45 min. The brain was immediately removed from the skull, blocked, photographed, and then stored in $30 \%$ buffered sucrose at $4^{\circ} \mathrm{C}$ until it sank. Frozen sections were cut in the coronal plane at 35 or $40 \mu \mathrm{m}$ in the PHA-L cases and at $50 \mu \mathrm{m}$ in the WGA-HRP cases. All sections were processed in the PHA-L cases, whereas a series of every fifth section was processed in the WGA-HRP cases. The remaining sections in the latter cases were stained for Nissl and parvalbumin to determine the cortical areal and laminar borders. Some of the PHA-L sections were also stained for Nissl after the PHA-L observation was completed, to determine the cortical areal and laminar borders. Transported PHA-L was visualized by the same procedure as that described in Saleem et al. (1993). The HRP reaction was performed according to the modified tetramethyl benzidine method described by Gibson et al. (1984).

Data analysis. The sections were observed with a light microscope under bright- and dark-field illumination. To examine the global distribution of labeling, labeled terminals and cell bodies in STS, TEO, and TE were first plotted onto enlarged camera lucida drawings of sections, which were later transformed into two-dimensional unfolded maps. Sections were usually sampled at $0.5 \mathrm{~mm}$ intervals in the WGA-HRP cases to make the unfolded maps, and the sampling interval was decreased to $0.25 \mathrm{~mm}$ when the distribution of labeling was sparse. In the PHA-L cases, sections were sampled at 0.42 or $0.48 \mathrm{~mm}$ intervals to make the unfolded maps. To examine the laminar distribution of labeling, the PHA-L-labeled terminals were observed with a $10 \times$ objective lens in all the sections containing labeling, and the labeled terminals were plotted with a camera lucida in every 4 th-12th section $(140-420 \mu \mathrm{m})$.

In PHA-L cases, most of the labeled segments were axon terminals with synaptic boutons, especially in the regions with dense labeling. Axon segments without boutons were observed around the border between the white matter and layer 6 but constituted only a small percentage of the labeled segments within the clustering of labeling in the gray matter.

Unfolded map. A series of coronal sections encompassing the full rostrocaudal extent of STS, TE, and TEO (Fig. $1 A$ ) were drawn using camera lucida. Layer IV was then traced on these drawings using Nomarsky optics or from adjacent Nissl- and parvalbumin-stained sections. The middle point of the fundus and the upper and lower lips of STS were marked on these camera lucida drawings (Fig. 1B, right). The absolute distances from the middle point of the fundus to the upper and lower lips of STS were measured in each section using a digital curvimeter placed over layer IV. The layer IV contour lines were straightened, and the positions of the upper and lower lips of STS were marked on the straight lines. These straight contour outlines were then aligned in parallel to produce a two-dimensional unfolded map of the entire STS (Fig. $1 B$, middle). The outline of STS served as reference points in the arrangement of the sections; i.e., the middle points of the fundus were aligned along the shape of STS taken from the photograph of the lateral view of the brain (Fig. $1 B$, top left). The other sulci, the AMTS, posterior middle temporal sulcus (PMTS), rhinal sulcus (RS), and occipitotemporal sulcus (OTS), were also unfolded. The absolute distances between the sulci were maintained along the vertical contour lines in the unfolded map. Because the contour lines were not stretched or shrunken in the unfolding process, the distortion of cortical area is minimal in and around STS and larger at positions near the rhinal sulcus and occipitotemporal sulcus. The unfolded maps dorsally cover cortical regions up to the dorsal lip of STS, except Figure 15, where more dorsal regions are covered up to the ventral lip of the sylvian fissure (SF). The ventral limits of the unfolded maps are the medial lips of the rhinal sulcus and occipitotemporal sulcus. The parahippocampal gyrus and the medial part of the entorhinal cortex are not shown in the unfolded maps.

The labeling was projected to layer 4 along the axes of cortical columns within the coronal sections and plotted on the unfolded maps. Because the cortical columns in the dorsal and ventral banks of STS are rostrally or caudally tilted from the coronal sections, there remains some misalignment. The points in the upper and lower layers within a single column are plotted to different positions on the unfolded map, which are rostrocaudally displaced, but because the angle between the STS and horizontal plane was up to $45^{\circ}$ given the cortical thickness of $2.5 \mathrm{~mm}$, this misalignment is estimated to be $<1.8 \mathrm{~mm}$.

Nomenclature. There are several different proposals for subdividing 

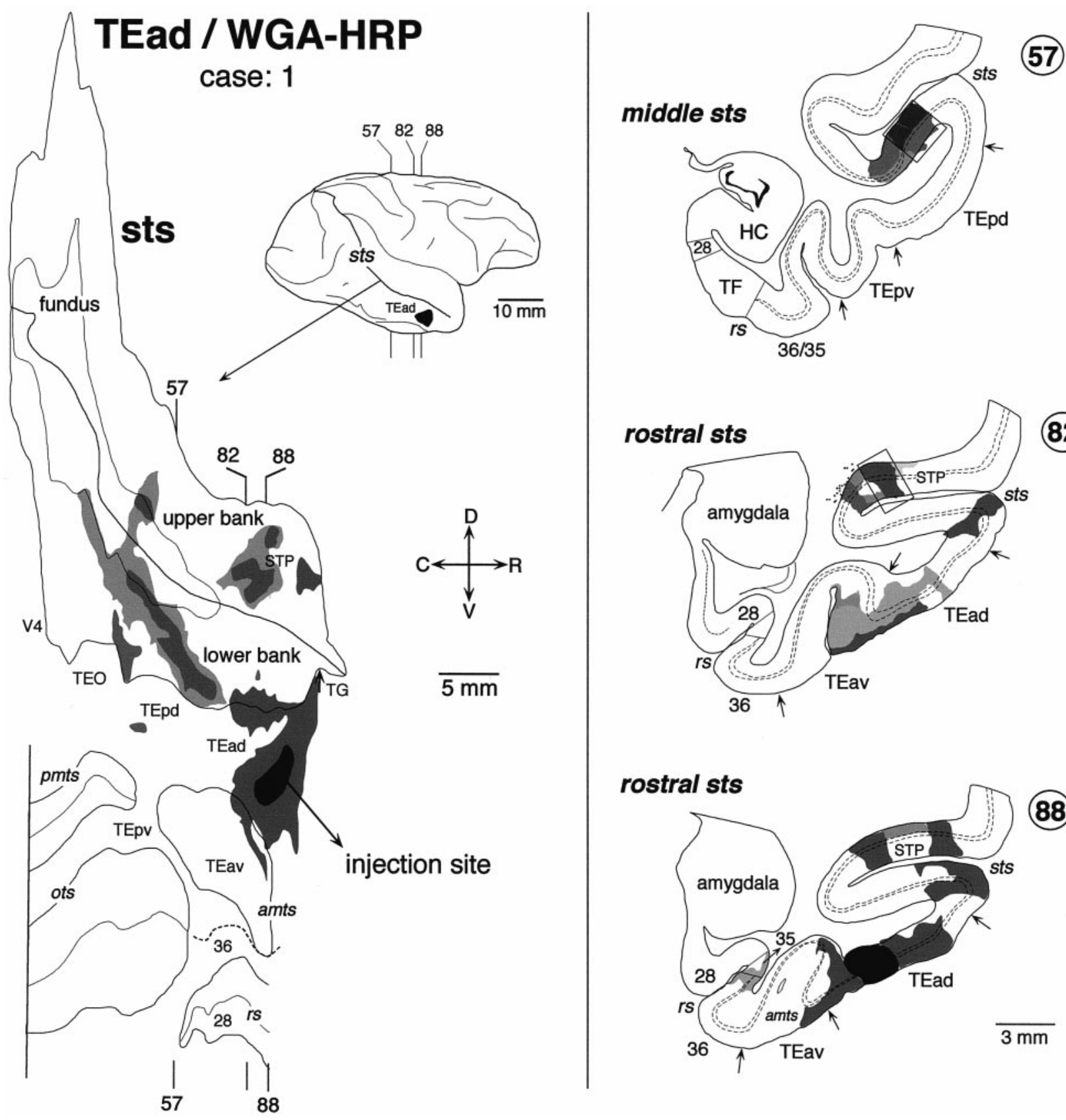

82

rostral sts

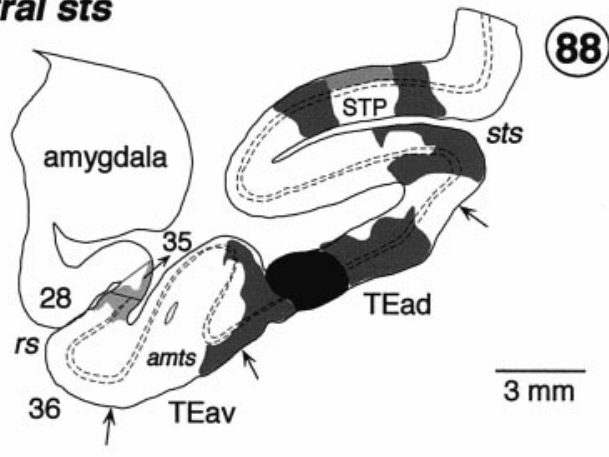

Figure 3. Distribution of labeled terminals and cell bodies in the two-dimensional unfolded map (left) and three representative coronal sections (right) after a WGA-HRP injection into TEad (case: 1). The darkest black region in the unfolded map and the lateral view of the brain indicate the extent of injection, whereas the gray regions indicate the distribution of labeled terminals and cell bodies. The darkness of the gray indicates the density of the distribution. The rostrocaudal levels of the illustrated coronal sections are indicated in the unfolded map as well as in the lateral view of the brain. The distribution of labeled terminals and cell bodies in the circumscribed regions in coronal sections (57 and 82$)$ are shown in the dark-field photomicrographs in Figure 7, $A$ and $B$. The brain regions posterior to the vertical lines in the unfolded map were not examined. Two groups of labeled terminals and cell bodies were found in STS: one group in the upper bank of the rostral STS and the other in the lower bank and fundus of the rostrocaudally middle part of STS. All other conventions are as in Figure 1.

TE in macaques based on cytoarchitectonic, connectional, or behavioral criteria (Seltzer and Pandya, 1978; Horel et al., 1987; Yukie et al., 1990; Felleman and Van Essen, 1991). With the anterior middle temporal sulcus as a landmark, Yukie and collaborators (Iwai and Yukie, 1988; Yukie and Iwai, 1988; Yukie et al., 1990) divided TE into four subregions: posterior dorsal (TEpd), posterior ventral (TEpv), anterior dorsal (TEad), and anterior ventral (TEav). We adopted this subdivision of TE, but we found previously that the border between TEad and TEav described by Yukie and collaborators (Iwai and Yukie, 1988; Yukie and Iwai, 1988; Yukie et al., 1990) corresponds to the cytoarchitectural border between TE2 and TE1 described by Seltzer and Pandya (1978) (Saleem and Tanaka, 1996). We therefore used the cytoarchitectural criterion used by Seltzer and Pandya (1978) to determine the border between TEad and TEav: layer V is less populated by neurons in TEad than in TEav (Saleem and Tanaka, 1996, their Fig. 2). The border thus determined was located at the lateral bank or lip of the AMTS at the rostrocaudal level and approached the STS as it continued further anteriorly. The lateral border of TEad was defined by the cytoarchitectural border between TE2 and TEm described by Seltzer and Pandya (1978). Thus, our TEad does not include TEm. 

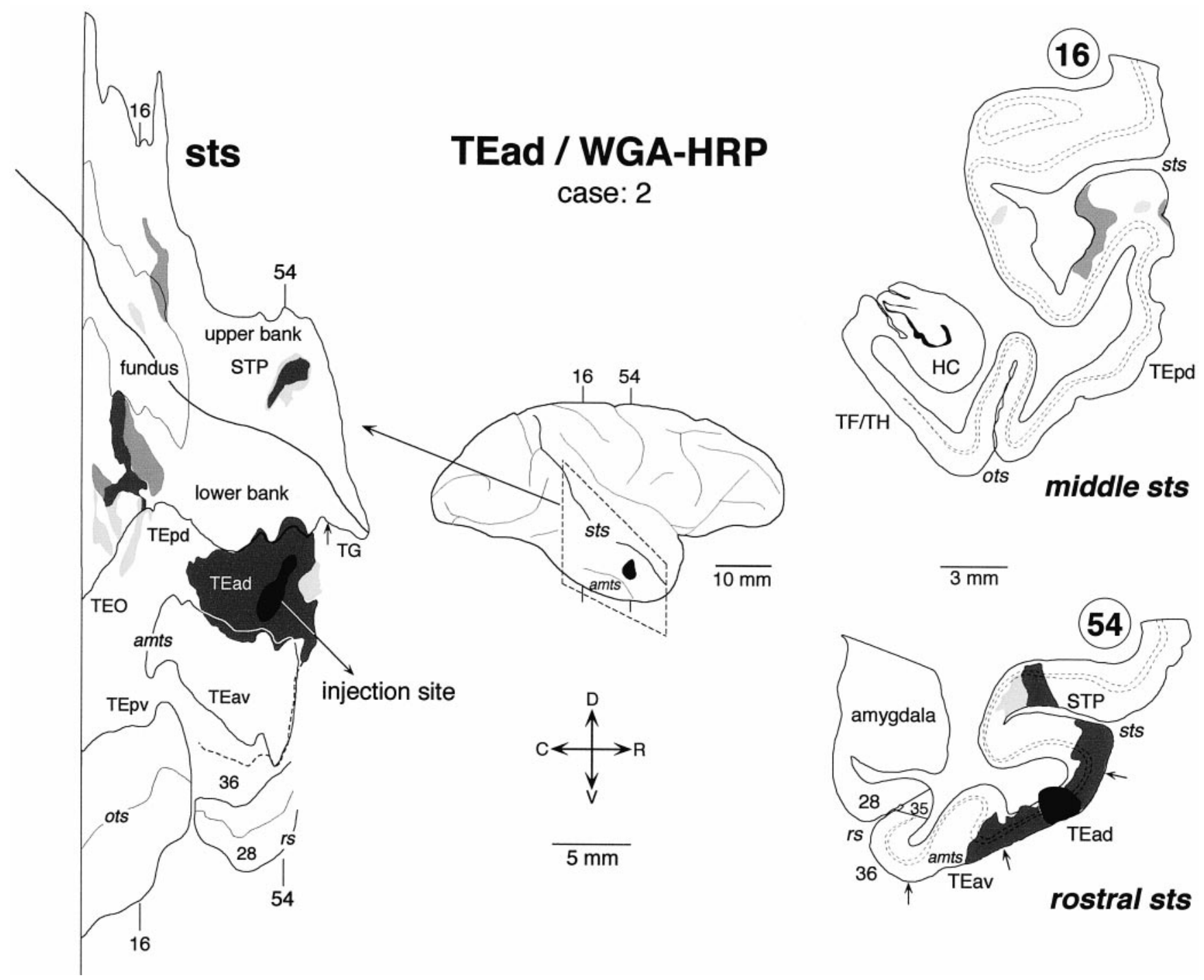

Figure 4. Distribution of labeled terminals and cell bodies after a WGA-HRP injection into TEad (case: 2). The rostrocaudal extent of the examined coronal sections is indicated by the broken lines in the lateral view of the brain. All other conventions are the same as in Figure 3.

Our definition of the border between TEav and the perirhinal cortex is similar to that of Amaral and colleagues (Suzuki and Amaral, 1994; Saleem and Tanaka, 1996). There was a clear separation between layers $\mathrm{V}$ and VI in TEav but not in area 36; differentiation of layer III into IIIA and IIIB was clearer in area 36 than in TEav, and the proportion of densely stained large pyramidal cells in layer $\mathrm{V}$ was greater in area 36 than in TEav (Saleem and Tanaka, 1996, their Figs. 2, 3). In addition, in the sections stained immunohistochemically for parvalbumin, there was a clear decrease in the density of staining at the border from TEav to area 36: the staining of both neurons and neuropil was lighter in area 36 than in TEav (Saleem and Tanaka, 1996, their Fig. 2B). The border between TEav and area 36 determined by the above-described criteria was located at a position one-third to one-half the distance from the medial lip of the AMTS toward the lateral lip of the rhinal sulcus at the caudal part corresponding to the caudal end of the rhinal sulcus, and it ran rostrally roughly parallel to the rhinal sulcus. In most cases in which the AMTS curved medially at its rostral end, the border was located at the medial lip of the rostral end of the AMTS.

Seltzer and Pandya (1978) differentiated several cytoarchitectonic areas in the rostral and middle parts of STS in the rhesus monkey (Macaca mulatta): TAa, TPO, and PGa in the upper bank, IPa in the fundus, and TEa and TEm in the lower bank. In the lower bank and fundus of the caudal part of STS, Seltzer and Pandya (1978) differentiated OAa, which corresponds to MT (Zeki, 1974; Van Essen et al., 1981) and FST (Desimone and Ungerleider, 1986). Subdivision in the upper bank of the caudal STS is similar to that of the rostral and middle STS. The upper bank and a part of the fundus of the rostral STS, which correspond to TAa, TPO, PGa, and IPa of Seltzer and Pandya (1978), have also been referred to as the STP (Bruce et al., 1981). Although we found it useful to describe our results in terms of the subdivisions proposed by Seltzer and Pandya (1978), we found it difficult to define these cytoarchitectonic borders in Japanese monkeys ( $M$. fuscata). Striking species differences between different macaques ( $M$. fuscata vs M. fascicularis) were also found in the size and relative positions of the central and surrounding auditory fields (Jones et al., 1995). We thus only indicate the upper bank, fundus, and lower bank in the unfolded maps of STS.

\section{RESULTS}

\section{Injection sites}

The PHA-L and WGA-HRP injections into TEad and TEav were well localized within TEad or TEav. The injection sites in the TEad cases were located at the middle between the lower lip of STS and the lateral lip of the anterior middle temporal sulcus (Figs. 2A, 3-5), whereas the injections sites in the TEav cases were located at the medial lip of the anterior middle temporal sulcus (Figs. 2B, 8-11). In the case in which WGA-HRP was injected at the border between TEav and area 36 (Figs. 2C, 13; 

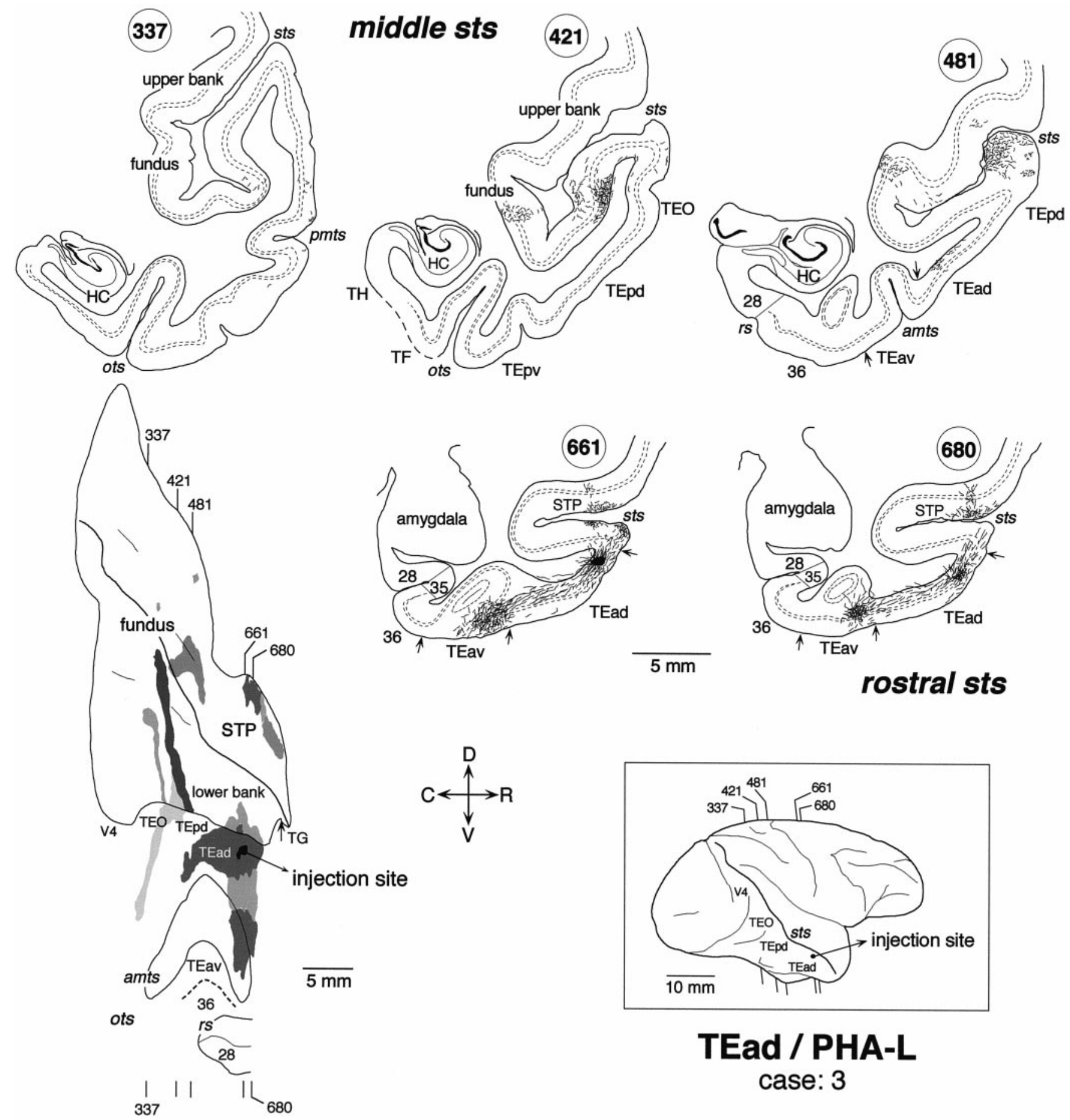

rostral sts

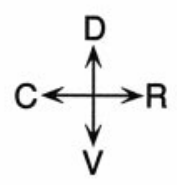

Figure 5. Distribution of anterogradely labeled terminals after a small PHA-L injection into TEad (case: 3). The gray shading in the unfolded map indicates the distribution of labeled terminals. The segments in the coronal sections represent labeled terminals and axon segments. The small filled region in the coronal section 661 indicates the PHA-L injection site. Other conventions are as in Figure 3.

TEav/36 border case), the injection did not cover the medial part of area 36 located within the rhinal sulcus. In the case in which WGA-HRP was injected into the caudal part of area 36 (Figs. 2D, 14), the injection covered the lateral lip of the rhinal sulcus, and its lateral border was $\sim 1.5 \mathrm{~mm}$ medial to the TEav/36 border. The PHA-L injections were circumscribed to small foci, which were $0.5-1.0 \mathrm{~mm}$ in width in the plane parallel to the cortical layers. They included all of the cortical layers, except in one TEad case (Fig. 5, case 3) in which the injection was restricted to layers 3-6. The WGA-HRP injections were larger (1.5-3.0 $\mathrm{mm}$ in width) and included all the cortical layers. Note that the shape of the injections in the flattened maps does not represent their exact shape because of distortion by the flattening procedure. 

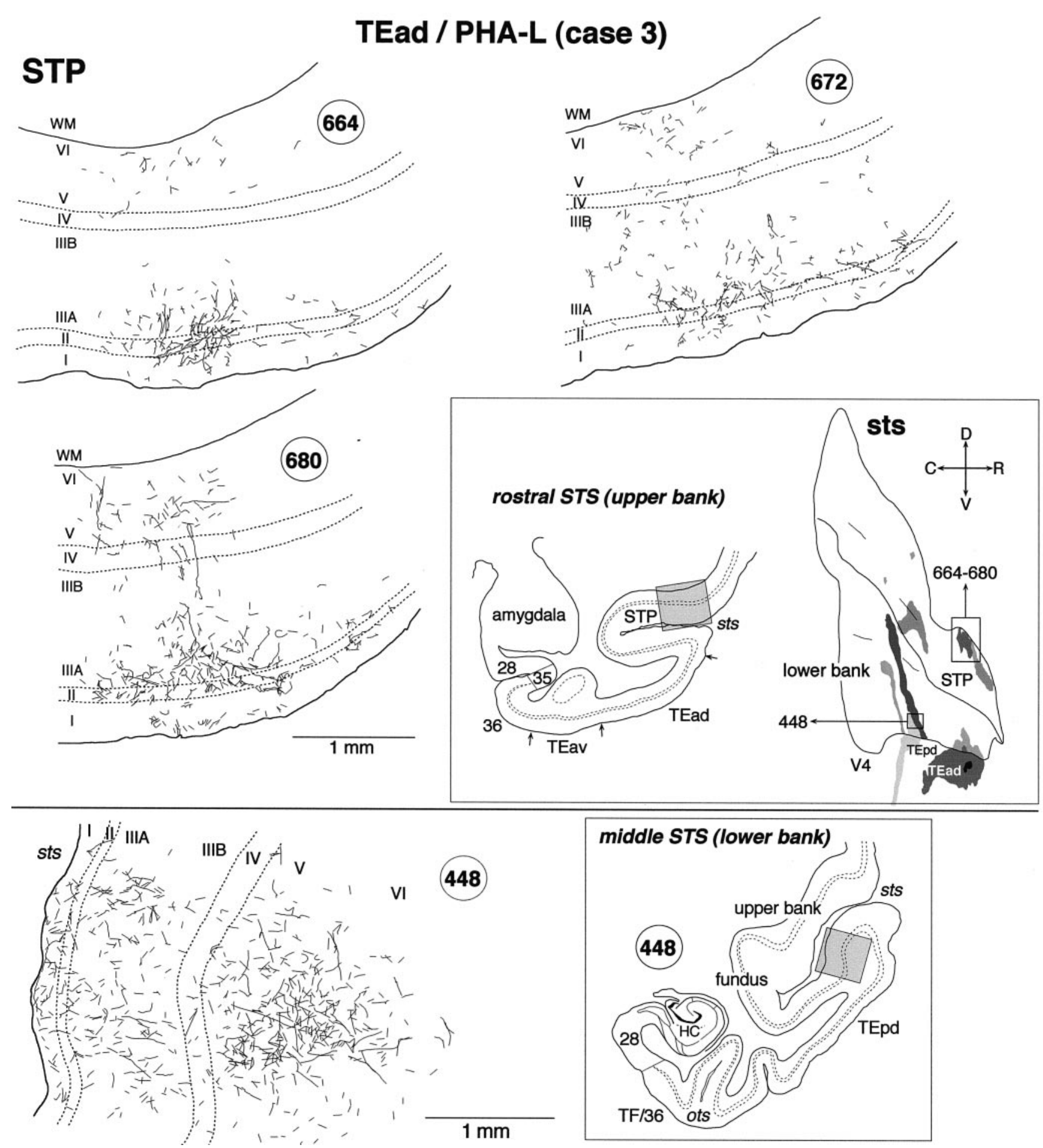

Figure 6. High magnification camera lucida drawings of patchy regions in STS that show the laminar distribution of PHA-L-labeled terminals in case 3. Top, Patches obtained from the upper bank of rostral STS (STP; sections 664, 672, and 680); bottom, a patch obtained from the lower bank of rostrocaudally middle STS (section 448). The positions of the patches are indicated by the gray shading in the coronal section drawings and by the rectangular boxes in the unfolded map.

\section{TEad Cases}

In all three TEad injection cases, the labeling in STS was mainly found in two groups of clusters; one in the upper bank of the rostral STS and the other in the lower bank and fundus of the rostrocaudally middle part of STS. The two groups of clusters do not overlap each other along the rostrocaudal axis. In the WGA-
HRP cases, the distribution of retrogradely labeled cell bodies roughly coincided with that of anterogradely labeled terminals in the plane parallel to the cortical surface. There was no cluster in STS that contained only labeled terminals or cell bodies in the TEad cases.

The distribution of labeling in the upper bank of the rostral 

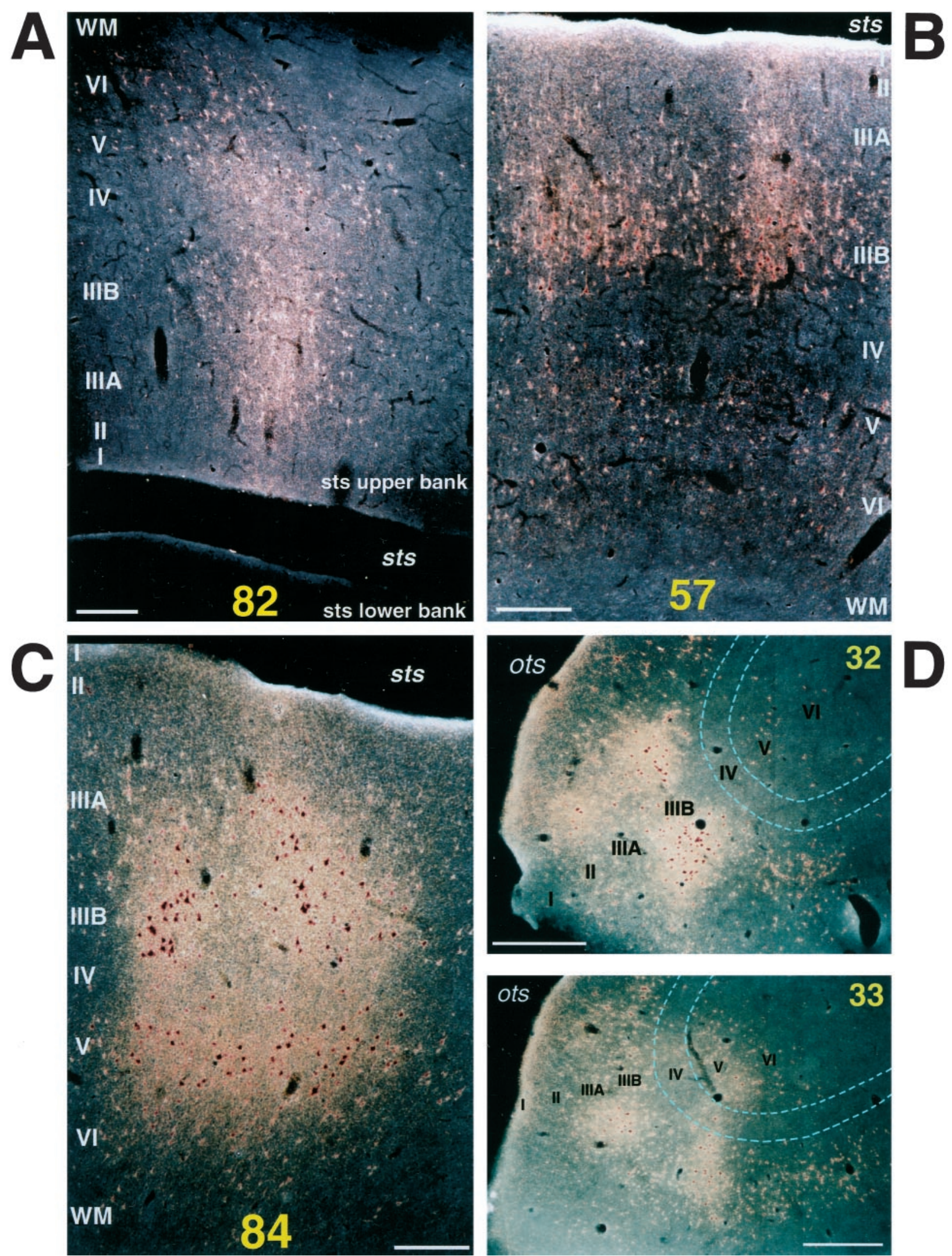

Figure 7. Dark-field photomicrographs showing the laminar distribution of labeled terminals and cell bodies in the rostral STS $(A, C)$ and the posterior regions $(B, D)$ after WGA-HRP injection into TEad $(A, B$, case 1$)$ and TEav $(C, D$, case 4$)$. $A$, A patch in section 82 obtained from the upper bank of the rostral STS (shown in Fig. 3); $B$, a patch in section 57 from the lower bank of the middle STS (shown in Fig. 3); $C$, a patch in section 84 from the lower bank of the rostral STS (shown in Fig. 8); D, two patches, one in section 32 and the other in section 33, obtained from the lateral lips of the occipitotemporal sulcus (shown in Fig. 8). Scale bars: $A-C, 0.25 \mathrm{~mm} ; D, 0.5 \mathrm{~mm}$. 

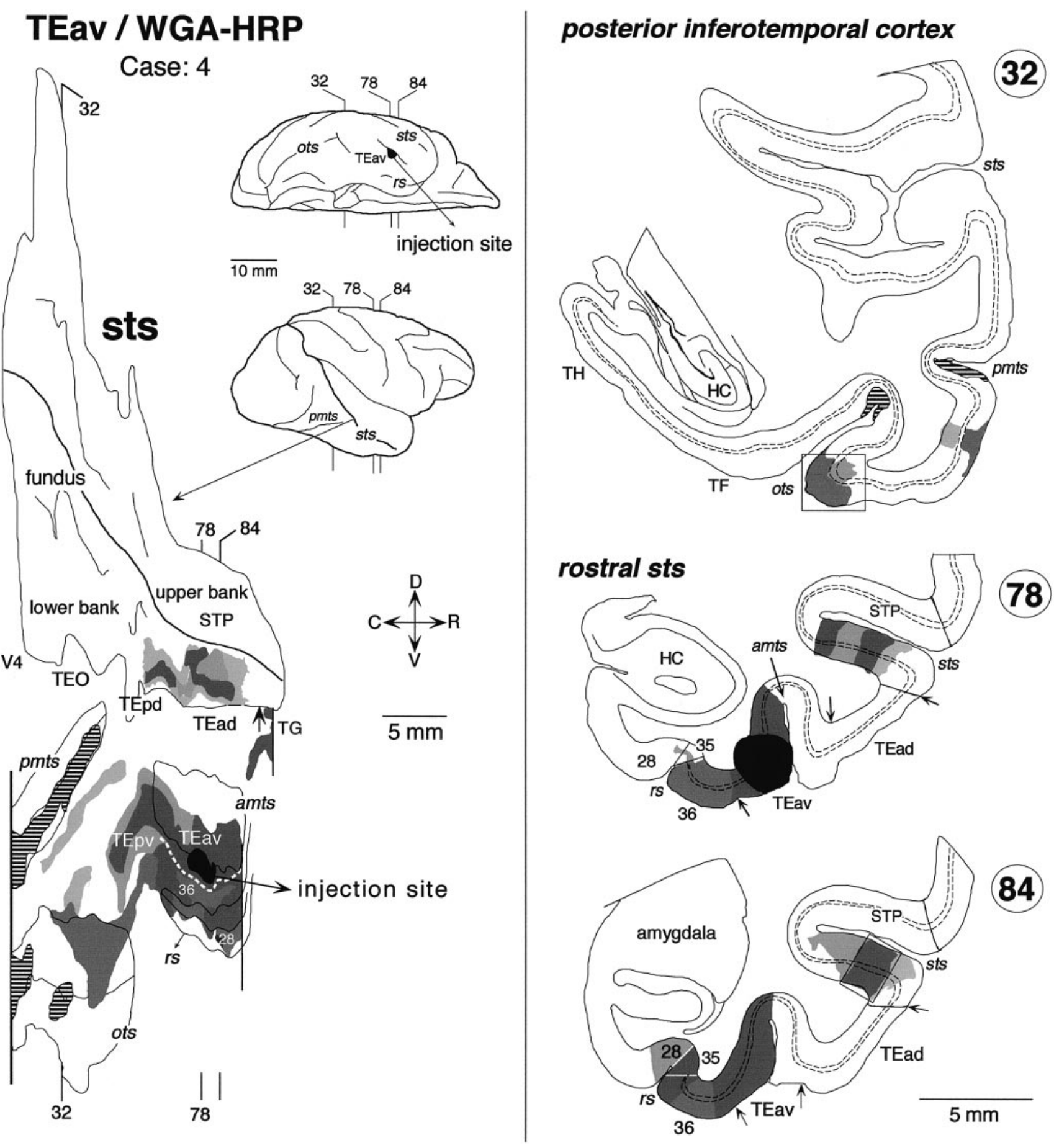

rostral sts

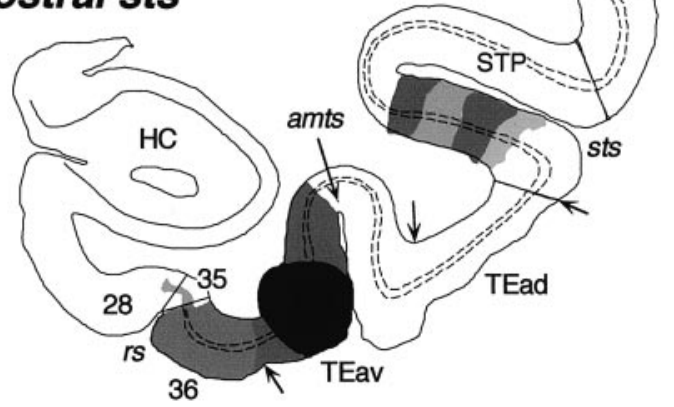

78

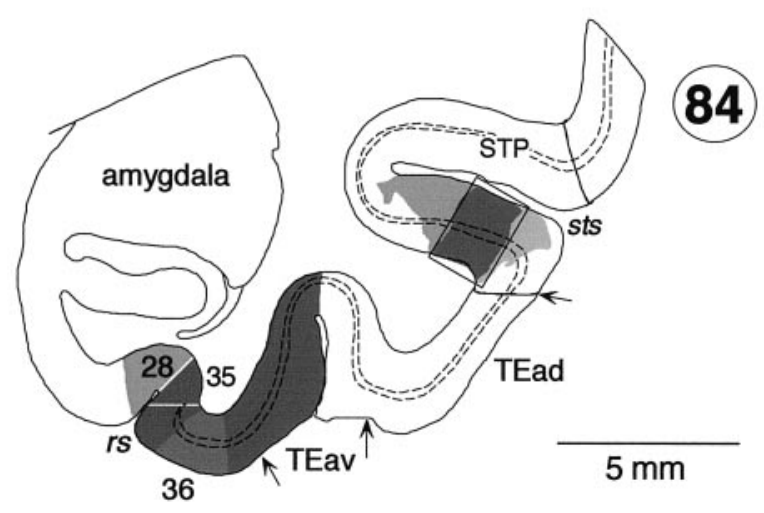

Figure 8. Distribution of labeled terminals and cell bodies after a WGA-HRP injection into TEav (Case: 4). The striped regions in the unfolded map (in pmts and ots) indicate the distribution of labeled terminals without labeled cell bodies. The labeled regions indicated by the boxes in coronal sections 84 and 32 are shown in the dark-field photomicrographs in Figure 7, $C$ and $D$, respectively. In STS, the distribution of labeling was limited to the rostral part of the lower bank. The brain regions beyond the vertical lines in the unfolded map were not examined. Other conventions are as in Figure 3.

STS was composed of two clusters in case 1 (Fig. 3) and one cluster in case 2 (Fig. 4). They occupied the middle depth of the upper bank, which mostly overlapped with TPO of Seltzer and Pandya (1978). The distribution of labeling in case 3 (Fig. 5) constituted a single cluster elongated rostrocaudally. This cluster in case 3 was located laterally closer to the upper lip of STS and seems to cover parts of both TPO and TAa.

The labeled terminals in the upper bank of the rostral STS were distributed in all the cortical layers (Figs. 3 and $7 A$, section 82 ; Fig. 6, section 680) or were found predominantly in layers 1-3 (Fig. 4, section 54; Fig. 6, sections 664 and 672) in both WGAHRP and PHA-L cases. The concentrations of labeled terminals in layer 2 and the upper part of layer 3 were the highest in the PHA-L case, whereas the quantitative comparison of labeled terminal concentrations was difficult in the WGA-HRP cases. The concentrations of labeled terminals in layer 1 were not higher 

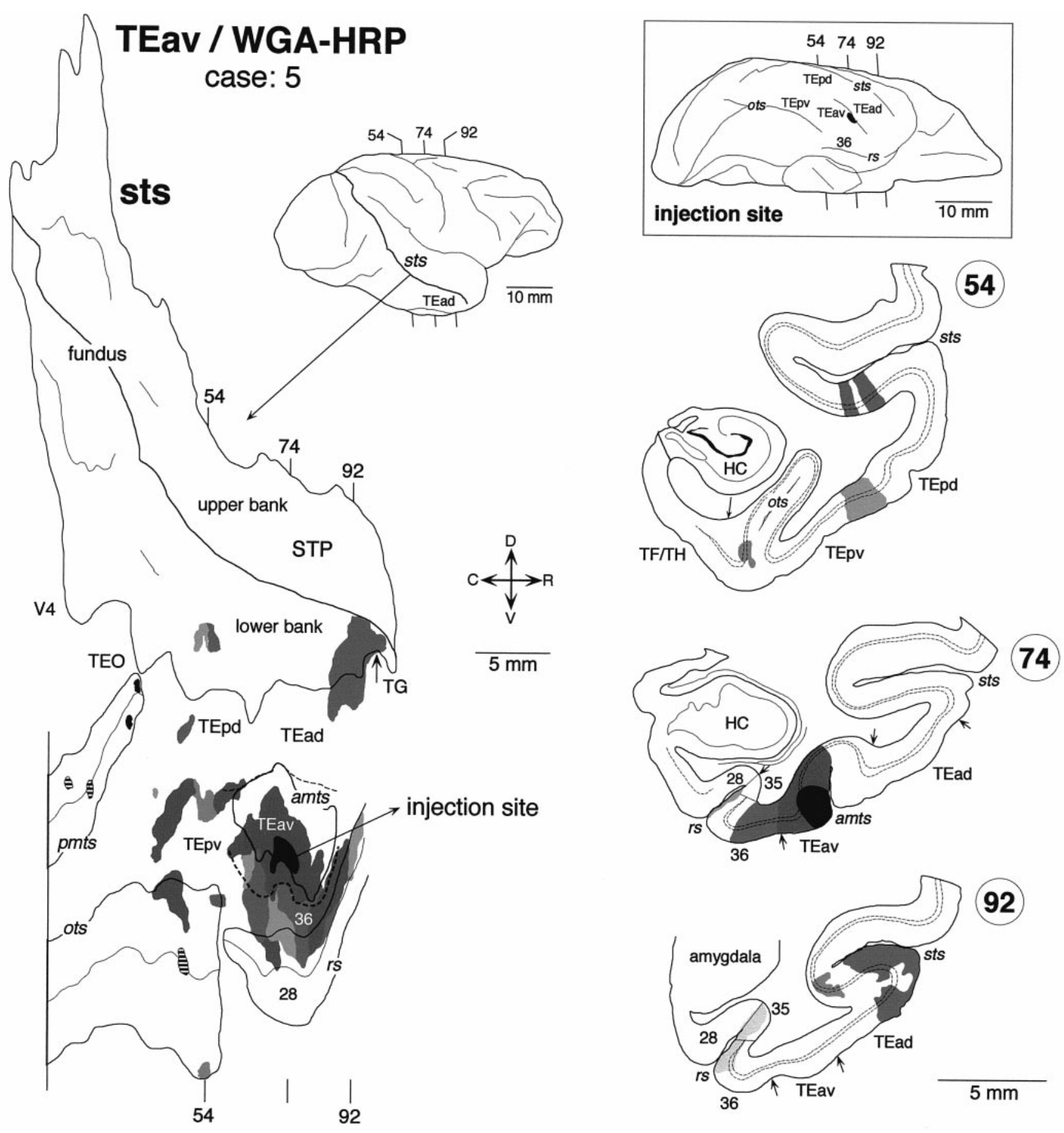

Figure 9. Distribution of labeled terminals and cell bodies after a WGA-HRP injection into TEav (case: 5). The small filled regions in the unfolded map (in pmts) indicate the distribution of labeled cell bodies without labeled terminals. The striped regions in the unfolded map (in pmts and ots) indicate the distribution of labeled terminals without labeled cell bodies. The brain regions posterior to the vertical line in the unfolded map were not examined.

than those in layer 2 and the upper part of layer 3. Cell bodies retrogradely labeled in the WGA-HRP cases were found equally in layers 2-3 and 5-6 (Figs. 3, 7A, section 82) or were restricted to layers 5-6. The confined distribution of labeled cell bodies in layers 5-6 was found in more rostral parts within the clusters.

The distribution of labeling in the lower bank and fundus of the rostrocaudally middle part of STS was composed of multiple clusters. Some of them were very elongated (Figs. 3, 5), whereas others had complex shapes (Fig. 4). Only a part of the elongation of clustering can be explained by the misalignment caused by the oblique cutting of cortical columns (see the Materials and Methods). Their exact positions along the rostrocaudal axis varied among the cases. They were located mostly at the TEp level in case 1 (Fig. 3), mostly at the TEO level in case 3 (Fig. 5), and around the borders between the TEp and TEO levels in case 2 (Fig. 4). The clusters in the fundus extended to the deepest part of the upper bank in all three cases. Therefore, it may be said that the distribution of labeling in the upper bank of STS was com- 

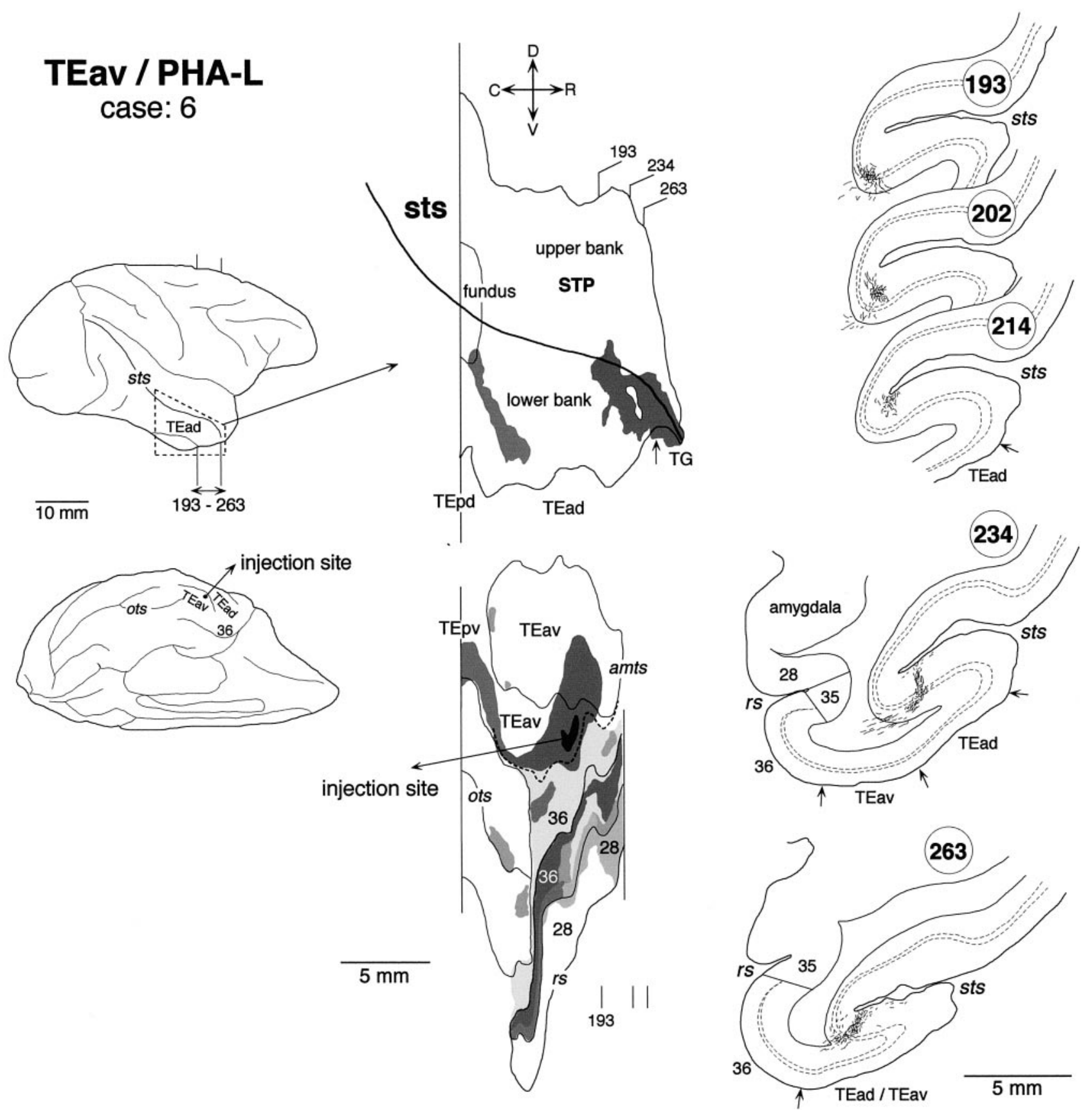

Figure 10. Distribution of anterogradely labeled terminals after a small PHA-L injection into TEav (case: 6). The brain regions caudal to the area circumscribed by broken lines in the lateral view of the brain were not examined. Other conventions are as in Figures 3 and 5.

posed of two groups: one in the rostral STS and the other in the middle STS. We do not favor this interpretation because the cluster in the middle STS was always located at the deepest part of the upper bank, whereas the cluster in the rostral STS was located at the middle or shallow part of the upper bank. The distribution in case 3 (Fig. 5) partially invaded FST in the fundus, but otherwise no labeling was found in MT, MST, FST, and V4t located in the caudal part of STS.

In WGA-HRP and PHA-L cases, the labeled terminals in the lower bank and fundus of the middle STS were distributed in both layers 1-3 and 5-6 (Figs. 3, 7B, section 57; Fig. 6, section 448), predominantly in layers 1-3 (Fig. 4, section 16; Fig. 5, section 481 ), or predominantly in layers 5-6 (Fig. 5, section 421). Layer
4 had none or only sparse distribution of labeled terminals. The concentrations of labeled terminals in layer 1 were as high or higher than those in layers 2 and 3. Retrogradely labeled cell bodies in the WGA-HRP cases were found in layers 2-3 and 5-6, with the densest distribution in layer $3 B$ (Fig. $7 B$ ). The labeled cell bodies in layers 2-3 appeared in dense clusters, whereas those in layers 5-6 were more evenly distributed (Fig. 7B).

Sparse labeling was observed in a small cluster in TEpd in case 1 (Fig. 3), and in an elongated cluster encompassing both TEO and TEpd in cases 2 and 3 (Figs. 4, 5). In all the cases, these distributions on the lateral surface were much less extensive than those in the adjoining regions in the lower bank of middle STS. The labeling resulting from the intrinsic connections around the 


\section{TEav / PHA-L case: 7}

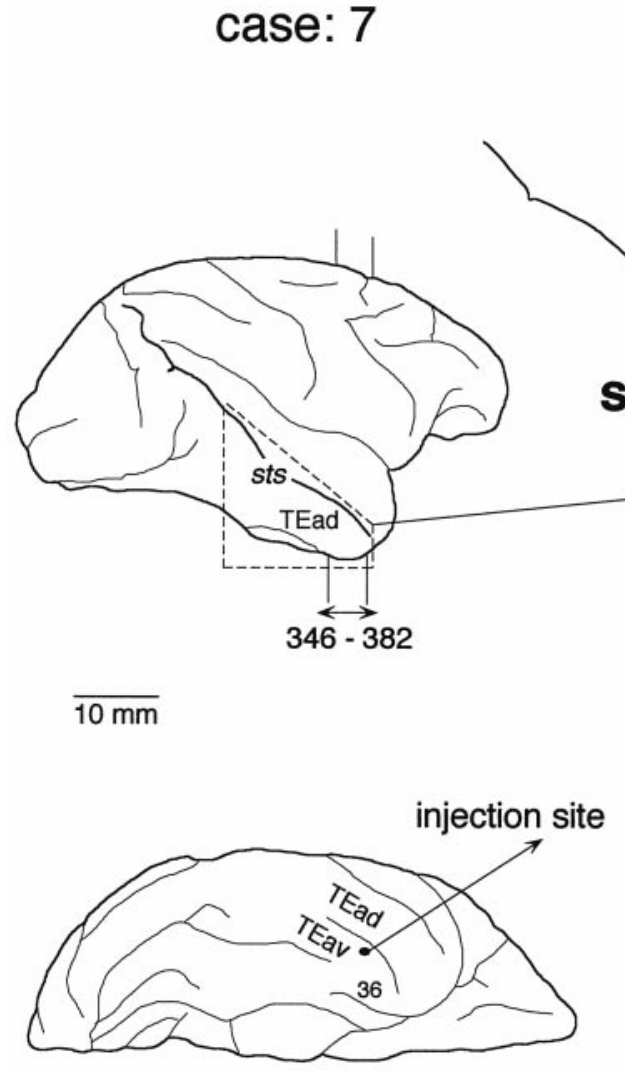

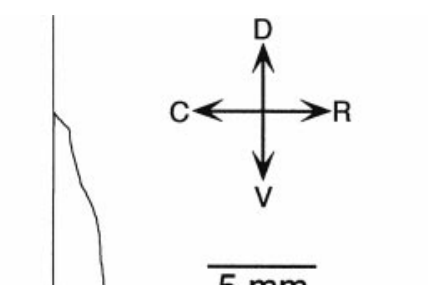
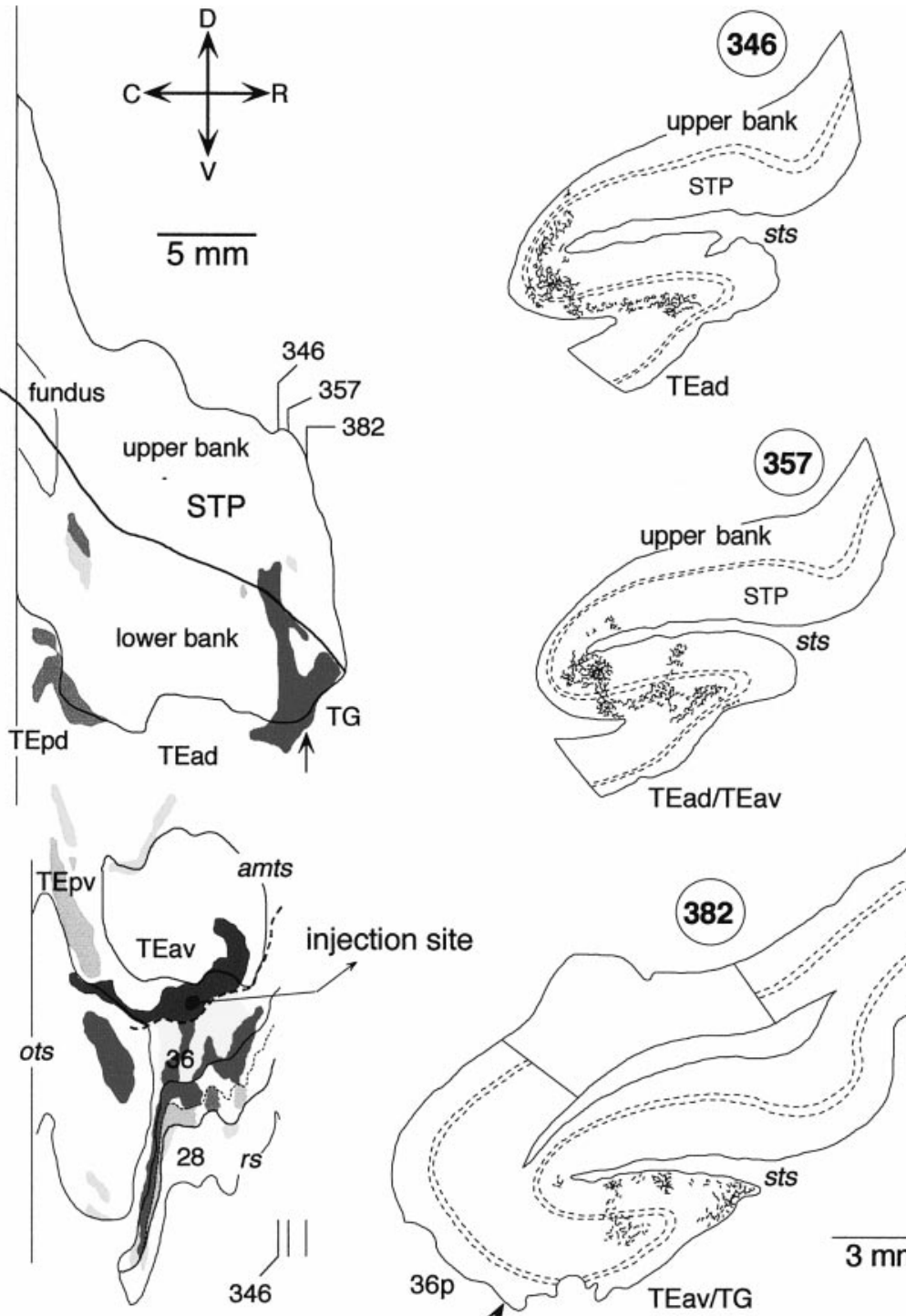

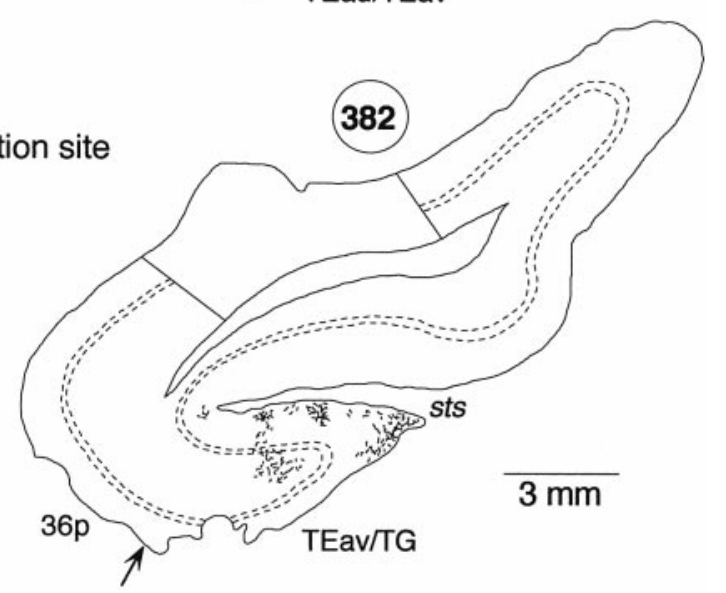

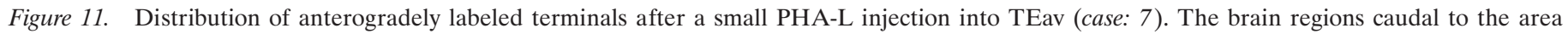
circumscribed by broken lines in the lateral view of the brain were not examined. All other conventions are the same as in Figures 3 and 5.

injection sites was extensive in all three cases, but they invaded the lower bank of STS only minimally. None of the cases showed labeling in TEpv or the ventral part of TEO.

\section{TEav cases}

True of all five TEav injection cases, the distribution of labeling in STS was limited to the lower bank and fundus of the rostral half of the sulcus. No labeling was found in the more caudal parts of STS. In the WGA-HRP cases, the distribution of retrogradely labeled cell bodies roughly coincided with that of anterogradely labeled terminals in the plane parallel to the cortical surface.

There was some variation in the detailed pattern of labeling distribution in the lower bank and fundus of STS. The distribution in cases 4 and 8 was composed of one large cluster, which extended in the lower bank of the rostral half of STS (Fig. 8) (case 8 is not shown). The rostrocaudal levels of the clusters corresponded to TEad and the anterior half of TEpd but did not invade TG. The distribution in cases 5, 6, and 7 was composed of two clusters (Figs. 9-11). One cluster, which was larger than the other, was located at the rostral end of STS and invaded TG in all three cases. The other, smaller cluster was located at the rostrocaudal level corresponding to TEpd in cases 5 and 7 (Figs. 9, 11) and the border between TEpd and TEad in case 6 (Fig. 10). The distribution of labeling slightly invaded the upper bank of STS in cases 6 and 7 (Figs. 10, 11), but not in cases 4, 5, and 8 (Figs. 8, 9).

In both WGA-HRP and PHA-L cases, the labeled terminals in STS were distributed in all the cortical layers (Figs. $7 C, 8$, section 84; Fig. 8, section 78; Fig. 9, section 54; Fig. 10, section 234; Figs. 10, 12, section 263; Fig. 11, sections 346, 357, and 382) with the highest density always in layer 2 and the upper part of layer 3 (Fig. 12). In some cases, the terminals appeared in the deep layers (5-6) and gradually moved to the upper layers (1-3) (e.g., sections 193, 202, and 214 in Fig. 10). This movement seems to be caused by the cutting of sections oblique to the axis of cortical columns (see Materials and Methods). The labeled terminals in layer 4 were as dense as those in layer 5 and the lower part of layer 3 (Fig. 12). The labeled terminals in layer 1 were sparser than 


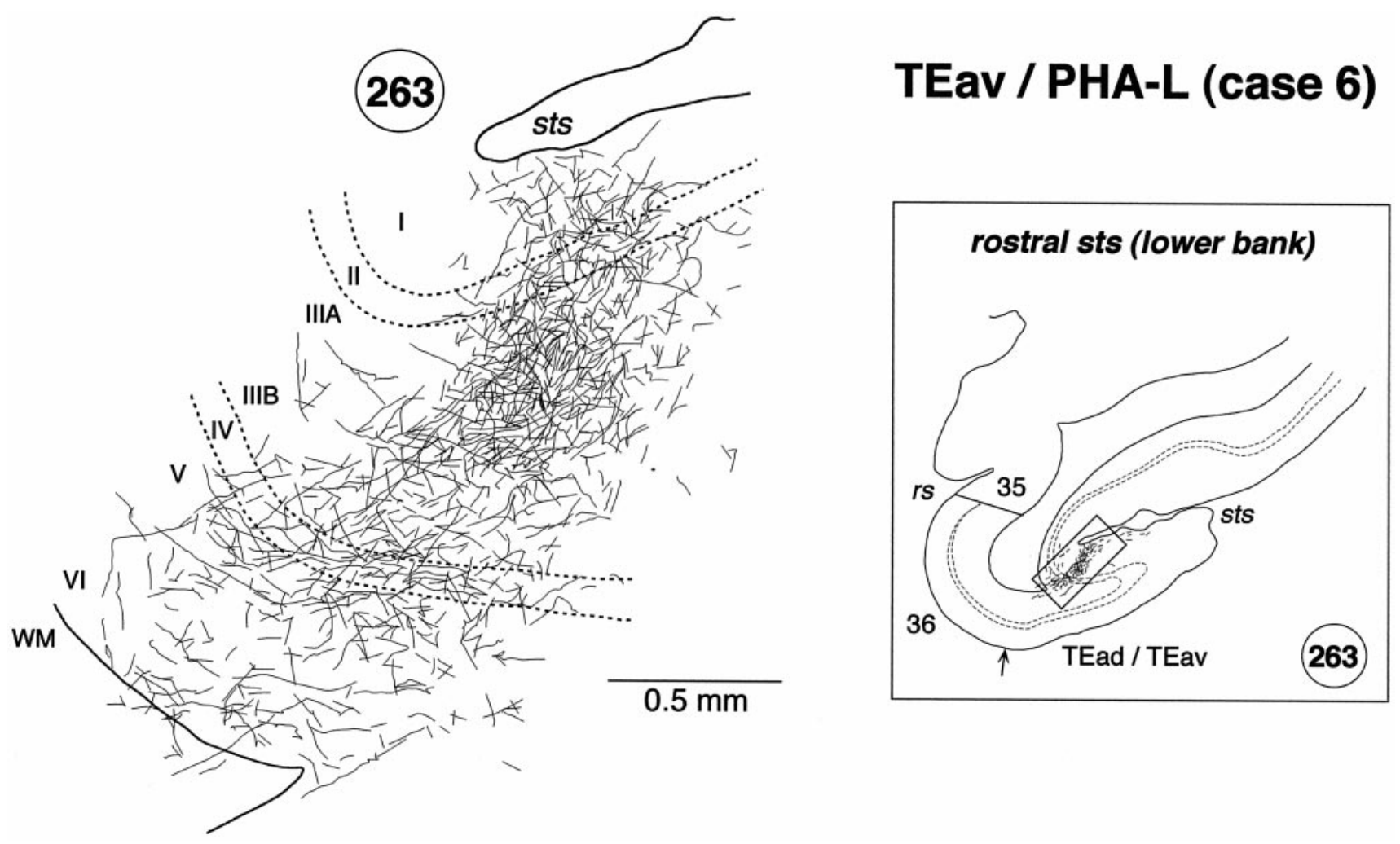

Figure 12. High magnification camera lucida drawing of a patchy region in the lower bank of the rostral STS showing the laminar distribution of PHA-L-labeled terminals in case 6 . The position of the patch in section 263 is indicated by a box in the inset on the right. Labeled terminals were distributed in all the cortical layers, but they were found more predominantly in layer 2 and the upper part of layer 3.

those in layer 2 and the upper part of layer 3. The cell bodies retrogradely labeled in the WGA-HRP cases were equally distributed in layers 2-3 and 5-6 (Figs. 7C, 8, section 84 ) but also found more in layers $2-3$ in some sections. The predominant distribution of labeled cell bodies in layers 2-3 was observed in the caudal and rostral edges of labeled zones. A few labeled cell bodies were occasionally found in layer 4 in some sections.

Clusters of labeling were also found in TEO and the TEpv. The most prominent clusters were located in the lateral bank and lateral lip of the OTS in all five cases (Figs. 8-11), and less prominent clusters were located at more lateral regions on the ventrolateral surface in most cases (Figs. 8, 9, 11). The labeling was also found in the PMTS in three WGA-HRP cases (Figs. 8, 9), whereas this sulcus was not included in the section cutting in the two PHA-L cases (Figs. 10, 11). Some of the clusters in the occipitotemporal sulcus and posterior middle temporal sulcus contained only labeled terminals (Figs. 8, 9, hatched regions) or cell bodies (Fig. 9, small filled regions in pmts). The clusters in the lateral bank and lip of the occipitotemporal sulcus were continuous with the distribution of labeling surrounding the injection within TEav in most cases (Figs. 8, 10,11). The distribution of labeling surrounding the injection sites also expanded to the perirhinal cortex in all five cases. We have described these projections from TEav to the perirhinal cortex in a previous paper (Saleem and Tanaka, 1996).

Labeled terminals were found in both layers $1-3$ and 5-6 in most of these posterior clusters (Figs. 7D, 8, section 32). Labeled terminals were also observed in layer 4 in some sections (Fig. 7D, section 33), but the concentrations in layer 4 were always lower than in layer 5 and the lower part of layer 3 .
Cell bodies retrogradely labeled in the WGA-HRP cases were found in both layers 2-3 and 5-6, with denser distribution in layers 2-3 (Fig. $7 D$, section 33), or largely confined to layers 2-3 (Fig. 7D, section 32).

\section{TEav/36 border case and area 36 case}

The injection sites in the above-described TEav cases were always located at the medial lip of the anterior middle temporal sulcus. TEav extends to the ventral surface medial to these injection sites (Saleem and Tanaka, 1996). Therefore, we decided to examine changes in labeling distribution as the injection site was moved medially into area 36 of the perirhinal cortex to see how the results varied with injection site. Two cases were specifically dedicated to this purpose: one with the injection of WGA-HRP into the TEav/36 border located at the intermediate position between the medial lip of the anterior middle temporal sulcus and the lateral lip of the rhinal sulcus (Figs. $2 C, 13$ ) and the other with a WGA-HRP injection into the caudal part of area 36 located at the lateral lip of the rhinal sulcus (Figs. 2D, 14).

In the TEav/36 border injection case, the distribution of labeling was confined to the lower bank of the rostral STS (Fig. 13), as in the TEav cases. The distribution of labeling was split into several small clusters with an overall rostrocaudal extent similar to that of the large clusters in case 4 (Fig. 8) and case 8 with TEav injections, except that there was one cluster in TG in the TEav/36 case. In the area 36 case there were again several clusters of labeling in the lower bank of the rostral STS as in the TEav and TEav/36 border cases, but there was also a distinctive cluster of labeling in the upper bank of the rostral end of STS (Fig. 14, 


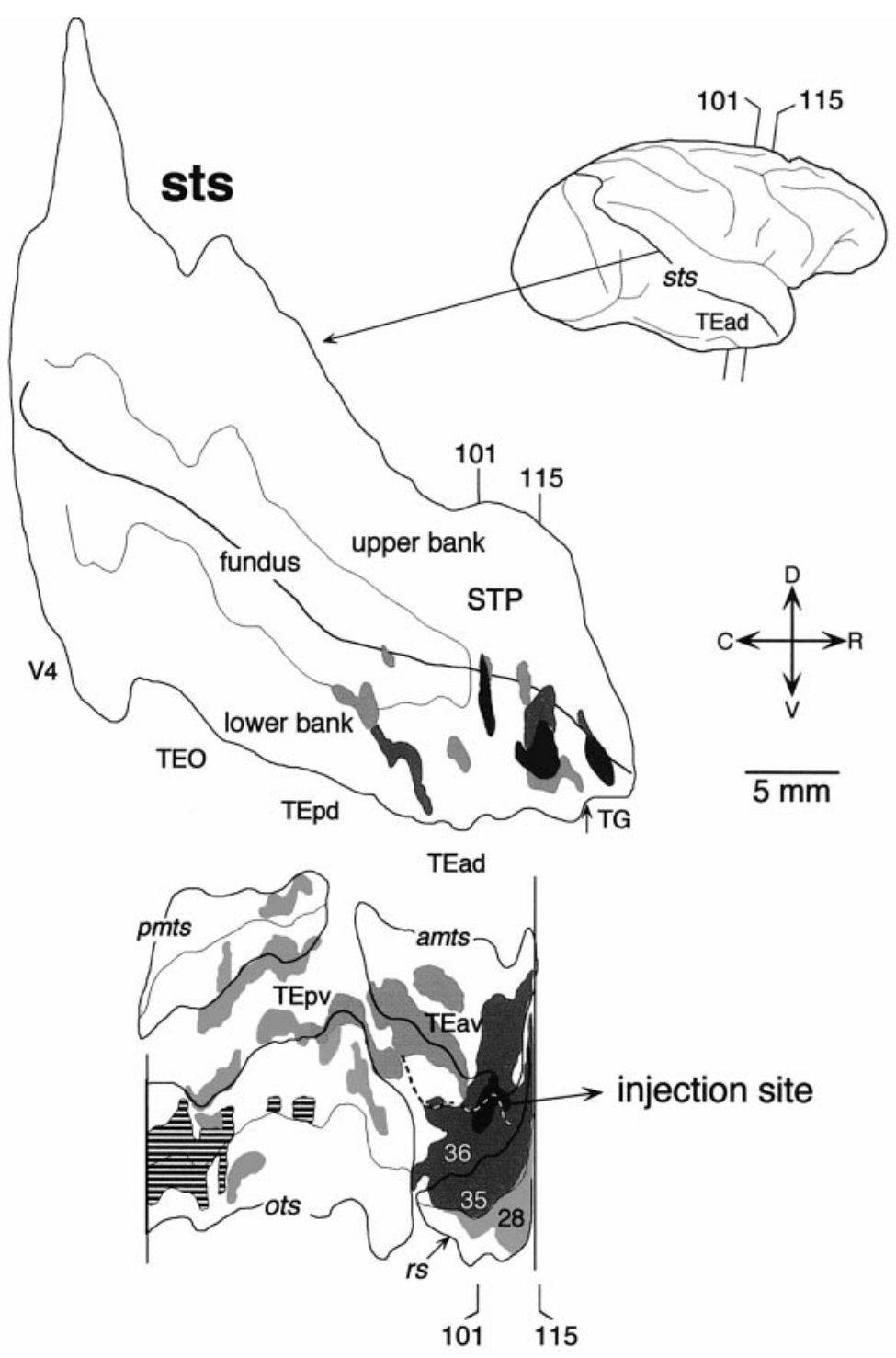

TEav-36 border / WGA-HRP case: 9
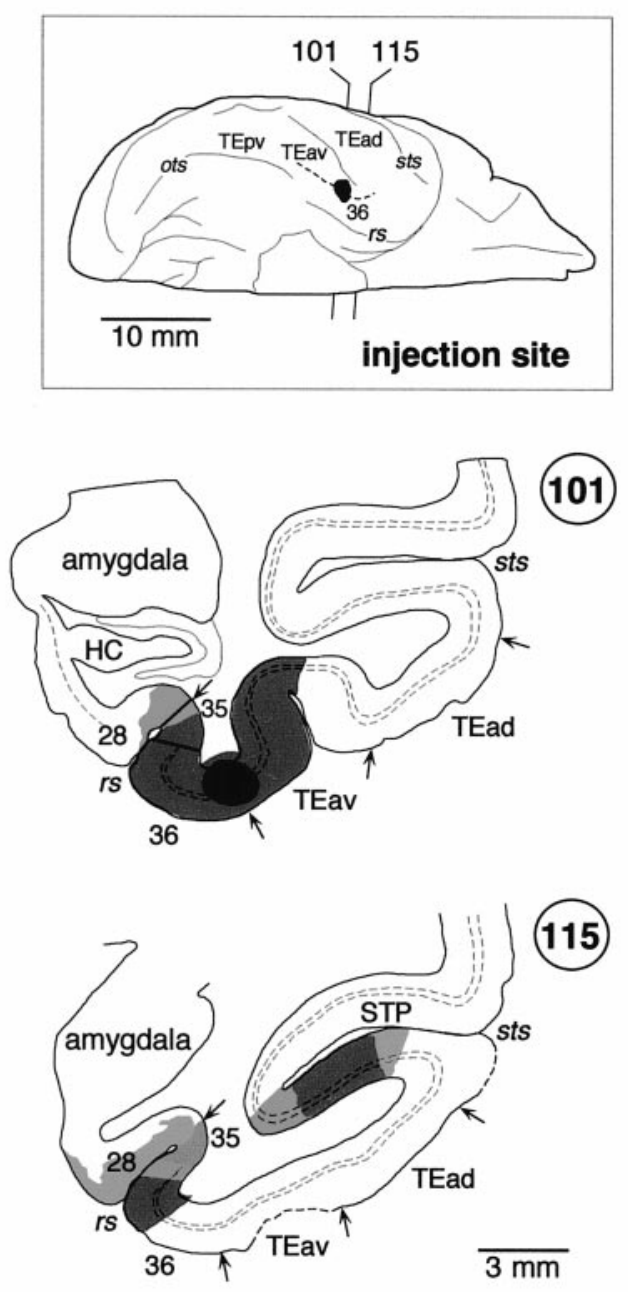

Figure 13. Distribution of labeled terminals and cell bodies after a WGA-HRP injection into the border between TEav and area 36 of the perirhinal cortex (case: 9). The striped regions in the unfolded map (ots) indicate the distribution of labeled terminals without labeled cell bodies. The brain regions anterior to the vertical line were not examined. Other conventions are the same as in Figure 3.

section 99). This part of STS did not contain labeling in both TEad and TEav cases.

In the TEav/36 border case, the labeled terminals were distributed in all cortical layers, and the labeled cell bodies were distributed in both layers 2-3 and 5-6 in the lower bank of the rostral STS (Fig. 13, section 115). In the area 36 case, the labeled terminals were mostly confined to layers $1-3$ and the labeled cell bodies to layers 2-3 in the lower bank of the rostral STS (Fig. 14, section 88). However, the labeled terminals were distributed in all cortical layers and the labeled cell bodies in both layers 2-3 and 5-6 of the upper bank of the rostral end of STS (Fig. 14, section 99).

\section{STP case}

We found that TEad was reciprocally connected with the upper bank of the rostral STS (STP), whereas TEav did not have connections with this STS region. To confirm these findings, we placed a WGA-HRP injection in the upper bank of the rostral STS in one case (Fig. 15, case 11). The injection was actually located at the upper lip of STS at the rostrocaudal level corresponding to TEad, and it covered parts of the superior temporal gyrus as well as the upper bank of STS. It covered all cortical layers. In addition to the dense distribution of labeling in STS and the superior temporal gyrus, there were discrete clusters of labeling in TEad (Fig. 15, section 108). There was also a sparse cluster of labeling within the medial bank of the RS, which corresponds to the lateral part of the entorhinal cortex (area 28). The labeling slightly invaded area 35 of the perirhinal cortex at the rostral end.

In the clusters found in TEad and the rhinal sulcus, the labeled terminals were distributed in all cortical layers, and the labeled cell bodies were distributed in both layers 2-3 and 5-6 or confined to layers $2-3$. Labeled terminals and cell bodies of various densities were found in the parahippocampal gyrus, insula, cingulate, and CA1 region of the hippocampus, which are not shown in Figure 15. No labeled terminals or cell bodies were found in TEav, more posterior inferotemporal regions (TEpd, TEpv, TEO), or area 36 of the perirhinal cortex. 

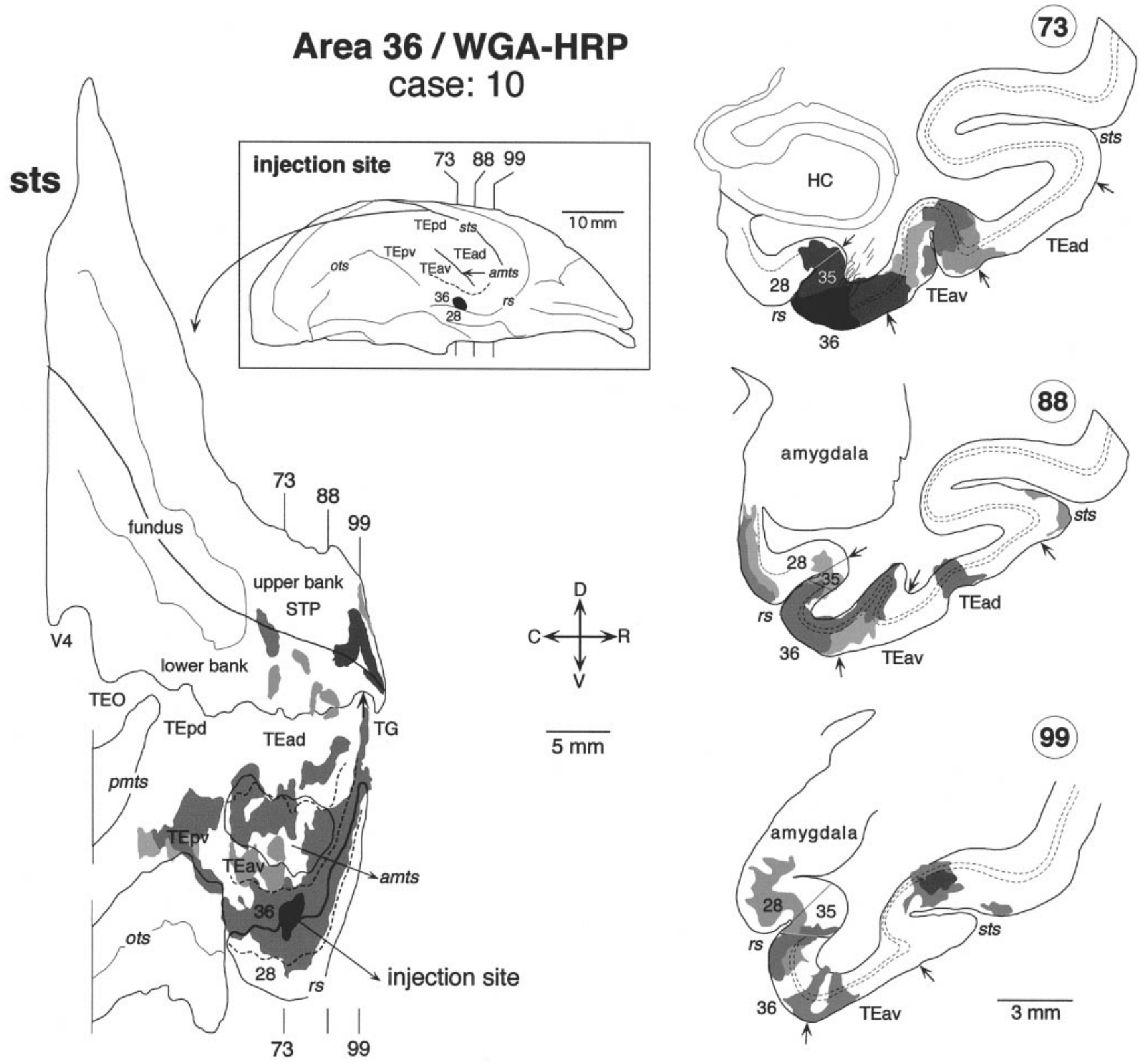

Figure 14. Distribution of labeled terminals and cell bodies after a WGA-HRP injection into the caudal part of area 36 of the perirhinal cortex (case: 10). For other conventions see Figure 3.

\section{DISCUSSION}

\section{Connections of TEad and TEav with rostral STS}

The present study reveals a dichotomy in the connections of TEad and TEav with the rostral STS. TEad connects with the upper bank of rostral STS, whereas TEav connects with the lower bank and fundus of rostral STS. The known differences in TEad and TEav connections are illustrated in Figure 16.

Our results challenge the classical view that the lower bank of rostral STS is connected with TE, whereas the upper bank of rostral STS is connected with the parietal, prefrontal, and superior temporal regions (Seltzer and Pandya, 1978, 1991, 1994; Baizer et al., 1991; Barnes and Pandya, 1992). However, our results are consistent with the results of Morel and Bullier (1990). They placed large injections of retrograde tracers into TEad and TEpd and found a patchy distribution of labeled cell bodies in the upper bank in addition to a continuous distribution in the lower bank of STS. There are two other previous studies that also found similar distribution of labeled terminals or cell bodies in STS after large injections of anterograde or retrograde tracers into TEad and/or TEpd (Baizer et al., 1991, their Figs. 2, 4, 7, and 12; Webster et al., 1991, their Fig. 17B), although the presence of labeling in the upper bank of STS was not emphasized in the text. The continuous labeling in the lower bank can be explained by the inclusion of the lower lip in their injections. The lower lip of STS, which corresponds to the TEm of Seltzer and Pandya (1978), was shown to be heavily connected with the middle depth of the lower bank of STS (Seltzer and Pandya, 1989b). We believe that the distribution of labeling in the lower bank after the large injections of tracers into TEad and/or TEpd in the previous studies was caused by the connections of TEm with the lower bank. 


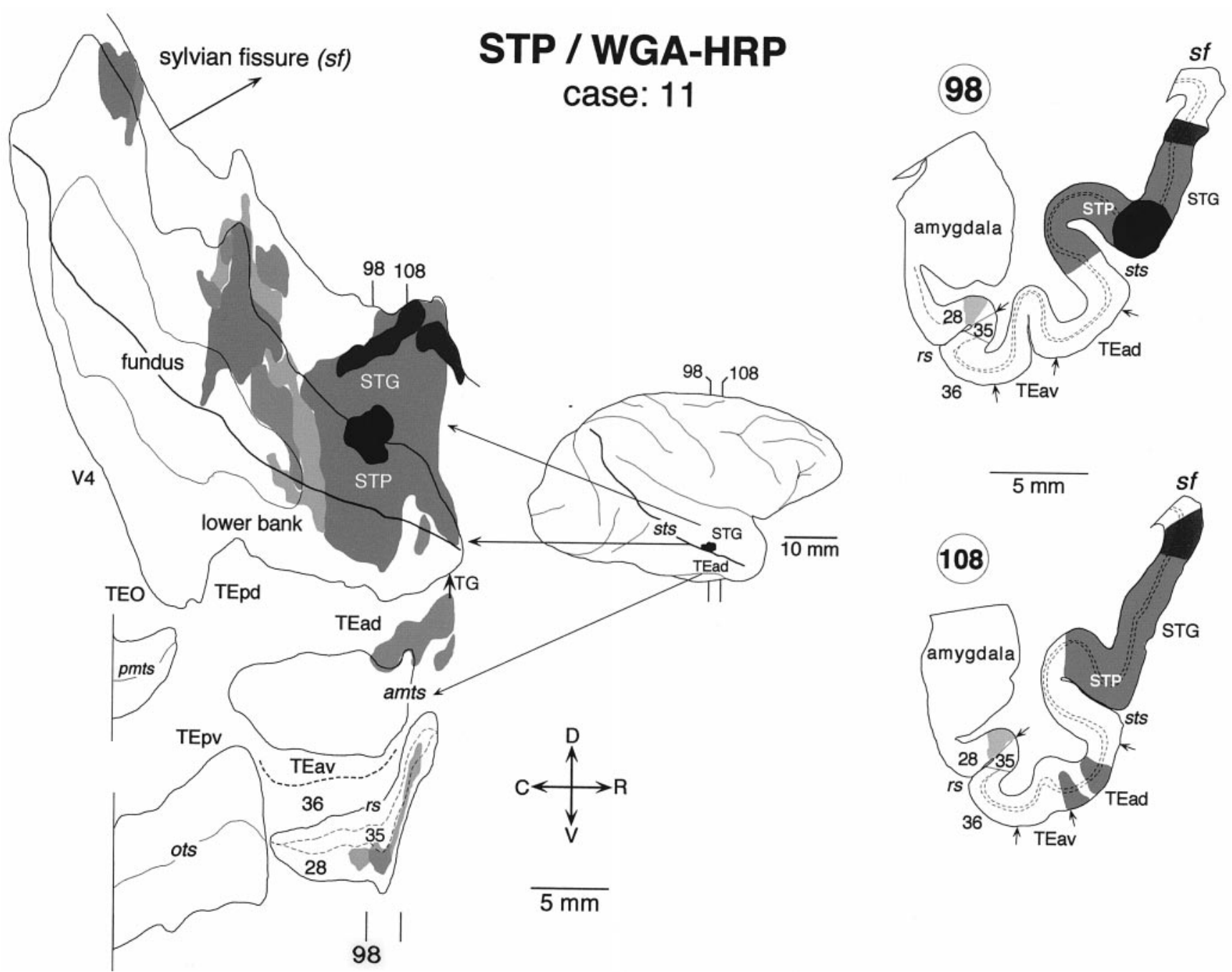

Figure 15. Distribution of labeled terminals and cell bodies after a WGA-HRP injection into the upper bank of rostral STS in the superior temporal polysensory area $(S T P)$ (case: 11). Labeling was found in TEad but not in TEav. Other conventions are as in Figure 3.

The present study also shows that TEad and TEav projections are distributed to all cortical layers in most parts of the terminal fields in the rostral STS. Cells projecting back to TEad and TEav were located in layers $2-3$ and $5-6$ in the rostral STS. These laminar patterns of labeled terminals and cell bodies are similar to those of the "lateral type" projections in sensory pathways (Rockland and Pandya, 1979; Maunsell and Van Essen, 1983; Felleman and Van Essen, 1991) and the projections between temporal and parietal, prefrontal and parietal, and prefrontal and temporal association cortical regions (Goldman-Rakic and Schwartz, 1982; Cavada and Goldman-Rakic, 1989; Seltzer and Pandya, 1989a, 1994; Andersen et al., 1990; Baizer et al., 1991; Webster et al., 1994).

\section{Connections of TEad and TEav with posterior areas}

The present study found that TEad is heavily connected with the lower bank of the rostrocaudally middle part of STS, whereas TEav is heavily connected with the lateral lip and lateral bank of the occipitotemporal sulcus, which correspond to TEpv or the ventral part of TEO. The laminar pattern of terminal distribution of the projections from TEad and TEav to these posterior regions are consistent with those of the "feedback type," and the laminar patterns of the cells projecting from the posterior regions to TEad and TEav are consistent with those of the "feedforward type," suggesting that TEad and TEav are higher than the posterior regions in the connectional hierarchy (Felleman and Van Essen, 1991).

These findings are consistent with the results of Martin-Elkins and Horel (1992) stating that large injections of WGA-HRP into the inferior temporal gyrus including parts of TEav and perirhinal cortex resulted in heavy labeling in both lateral and medial banks of the posteriorly located occipitotemporal sulcus. Together with previous findings of serial connections from central V4 to TEad through the dorsal TEO and TEpd, Martin-Elkins and Horel (1992) proposed that the ventral and dorsal parts of the anterior inferior temporal cortex receive visual inputs from the occipital cortex through separate pathways. This scheme is supported by Yukie et al. (1992). We modify this scheme with our finding of visual afferent pathways to TEad from the lower bank of middle STS. Our results do not deny the presence of afferent pathways along the lateral surface. The distribution of labeling surrounding the TEad injections always expanded in the posterior direction on the lateral surface. However, the dense distribution of labeling extended more posteriorly along the lower bank of STS than along the lateral surface. The pathway through the 


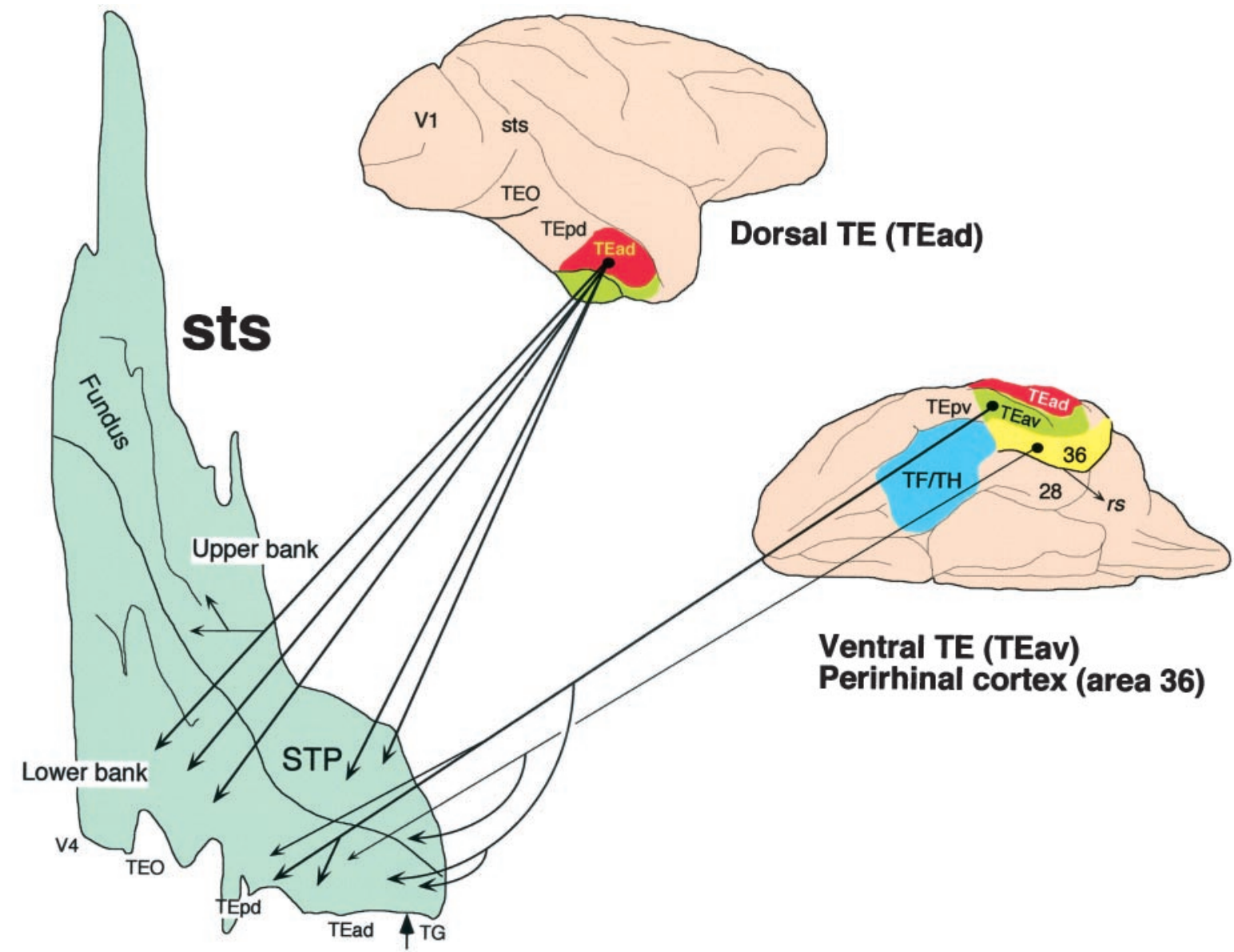

\section{Superior temporal sulcus}

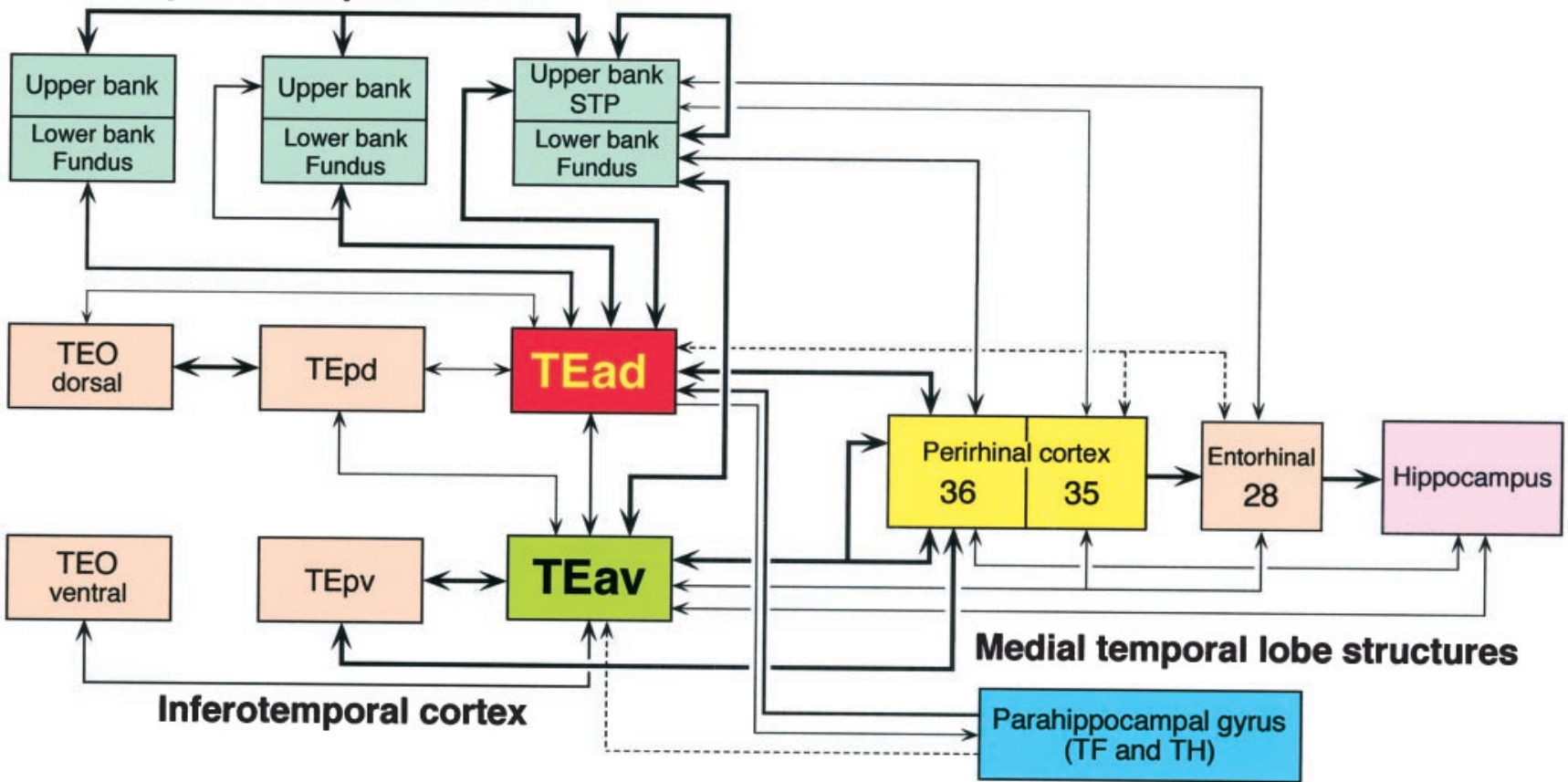

Figure 16. Top, Summary diagram of the projections from TEad, TEav, and area 36 of the perirhinal cortex to different regions in STS. The heavy solid lines denote dense projections, and thin solid lines denote moderate to sparse projections. Arrowhead at the rostral end of the flattened map indicates the border between TG and TEad. Bottom, Connections of TEad and TEav with STS, the posterior inferotemporal cortex, and the medial temporal lobe structures based on the present and previous studies (Yukie et al., 1990; Saleem and Tanaka, 1996; Saleem et al., 1996; Saleem and Hashikawa, 1998). The heavy solid lines indicate dense projections, the thin solid lines moderate to sparse projection, and the dashed lines very sparse projections. 
lower bank of STS may pass visual inputs to TEad more directly than the pathway along the lateral surface.

\section{Functional significance}

The upper bank of the rostral STS is known to be polymodal because it receives converging projections from the parietal, prefrontal, and superior temporal regions (Seltzer and Pandya, 1978, 1989a; Selemon and Goldman-Rakic, 1988; Boussaoud et al., 1990; Seltzer et al., 1996) and because it contains cells responsive to visual, auditory, or tactile stimuli, and cells responsive to more than one sensory modality (Desimone and Gross, 1979; Bruce et al., 1981; Baylis et al., 1987; Mistlin and Perrett, 1990). The upper bank of rostral STS corresponds to the anterior part of the STP of Bruce et al. (1981). Many of the visual responses in the anterior STP are sensitive to motion (Bruce et al., 1981; Baylis et al., 1987), especially to movements of the human body (Perrett et al., 1985; Oram and Perrett, 1994, 1996) and optic flow (Anderson and Siegel, 1999).

A possible function of the projection from the anterior STP to TEad is to provide the spatial context of objects to the analysis of object images in TEad via anterior STP afferents from parietal and prefrontal regions. The responses to optical flow support the idea that the anterior STP is involved in processing of spatial information. TEad may also receive spatial information from the parahippocampal gyrus: there are moderate to strong projections from the parahippocampal gyrus to TEad but not to TEav (Webster et al., 1991; Saleem et al., 1996). Another possible function of the projection from the anterior STP is to provide TEad with contextual information originating in audition and somatosensation. Although TEad is known to be purely visual (Desimone and Gross, 1979; Baylis et al., 1987), discharges in TEad cells can be triggered by behaviorally significant auditory and somatosensory stimuli (Iwai et al., 1987; Gibson and Maunsell, 1997; Hasegawa and Tanaka, 1998). This auditory and somatosensory influence may originate in the anterior STP. Other potential sources include the prefrontal cortex, perirhinal cortex, and parahippocampal gyrus. The projections from TEad may provide visual object information to the anterior STP, where the integration of visual object and motion information takes place (Perrett et al., 1985; Oram and Perrett, 1994, 1996).

The afferents to the lower bank of the rostral STS are less convergent than those to the upper bank. More caudal parts of the lower bank of STS (Seltzer and Pandya, 1989b), TEO on the lateral surface (Webster et al., 1991; Distler et al., 1993), and parts of TE (Seltzer and Pandya, 1978; Webster et al., 1991) project to the lower bank of rostral STS. The present study found that TEav, but not TEad, projected to this STS region. We also found that the connections of TEav were stronger with the lower bank of rostral STS than with TEad, although TEad is closer to the injection sites than the lower bank of rostral STS along the cortical surface. This suggests that the lower bank of rostral STS is distinctive from TEad. Cells in the lower bank of rostral STS have been reported to be purely visual (Desimone and Gross, 1979; Baylis et al., 1987). Most respond strongly to stationary stimuli, although Perrett et al. (1989) found cells selectively responding to the view of particular hand actions. H. Tanaka and I. Fujita (personal communication) found that most cells throughout the whole depth of the lower bank selectively respond to complex features of object images just as cells in TEad and TEpd do. However, a recent abstract of Janssen et al. (1999) reported that $67 \%$ of cells in the lower bank of STS selectively responded to disparity-defined three-dimensional shapes, whereas $13 \%$ of cells in the lateral surface did. The reciprocal connections between the lower bank of rostral STS and TEav may mediate interactions between the three-dimensional- and two-dimensional-based analyses of object images.

We have suggested previously that TEav is more involved in recognition memory than TEad because we found that TEav more strongly projected to the perirhinal cortex than did TEad (Saleem and Tanaka, 1996) (see Horel et al., 1987; Buckley et al., 1997) and that TEav, but not TEad, projected to the medial basal nucleus of the amygdala (Cheng et al., 1997). Other unique projections of TEav are directed to the nucleus accumbens of the ventral striatum and the lateral basal nucleus of the amygdala (Cheng et al., 1997), both considered important in relating emotion to motor behavior (Kalivas and Nakamura, 1999). It is thus possible that TEav is more involved in direct emotional behaviors related to objects, whereas TEad participates in context-based and strategic behaviors related to objects.

\section{REFERENCES}

Andersen RA, Asanuma C, Essick G, Siegel RM (1990) Corticocortical connections of anatomically and physiologically defined subdivisions within the inferior parietal lobule. J Comp Neurol 296:65-113.

Anderson KC, Siegel RM (1999) Optic flow selectivity in the anterior superior temporal polysensory area STPa of the behaving monkey. J Neurosci 19:2681-2692.

Baizer JS, Ungerleider LG, Desimone R (1991) Organization of visual inputs to the inferior temporal and posterior parietal cortex in macaques. J Neurosci 11:168-190.

Barnes CL, Pandya DN (1992) Efferent cortical connections of multimodal cortex of the superior temporal sulcus in the rhesus monkey. J Comp Neurol 318:222-244.

Baylis GC, Rolls ET, Leonard CM (1987) Functional subdivisions of the temporal lobe neocortex. J Neurosci 7:330-342.

Boussaoud D, Ungerleider LG, Desimone R (1990) Pathways for motion analysis: cortical connections of the medial superior temporal and fundus of the superior temporal visual areas in the macaque. J Comp Neurol 296:462-495.

Bruce C, Desimone R, Gross CG (1981) Visual properties of neurons in a polysensory area in superior temporal sulcus of the macaque. J Neurophysiol 46:369-384.

Buckley M, Gaffan D, Murray EA (1997) A functional doubledissociation between two inferior temporal cortical areas: perirhinal cortex vs middle temporal gyrus. J Neurophysiol 77:587-598.

Cavada C, Goldman-Rakic PS (1989) Posterior parietal cortex in rhesus monkey: I. Parcellation of areas based on distinctive limbic and sensory corticocortical connections. J Comp Neurol 287:393-421.

Cheng K, Saleem KS, Tanaka K (1997) Organization of corticostriatal and corticoamygdalar projections arising from the anterior inferotemporal area TE of the macaque monkey: a phaseolus vulgaris leucoagglutinin study. J Neurosci 17:7902-7925.

Desimone R, Gross CG (1979) Visual areas in the temporal cortex of the macaque. Brain Res 178:363-380.

Desimone R, Ungerleider LG (1986) Multiple visual areas in the caudal superior temporal sulcus of the macaque. J Comp Neurol 248:164-189.

Distler C, Boussaoud D, Desimone R, Ungerleider LG (1993) Cortical connections of inferior temporal area TEO in macaque monkeys. J Comp Neurol 334:125-150.

Felleman DJ, Van Essen DC (1991) Distributed hierarchical processing in the primate cerebral cortex. Cereb Cortex 1:1-47.

Gerfen CR, Sawchenko PE (1984) An anterograde neuroanatomical tracing method that shows the detailed morphology of neurons, their axons and terminals: immunohistochemical localization of an axonally transported plant lectin, Phaseolus vulgaris leucoagglutinin (PHA-L). Brain Res 290:219-238.

Gibson AR, Hansma DI, Houk JC, Robinson FR (1984) A sensitive low artifact TMB procedure for the demonstration of WGA-HRP in the CNS. Brain Res 298:235-241.

Gibson JR, Maunsell JHR (1997) Sensory modality specificity of neural activity related to memory in visual cortex. J Neurophysiol 78: 1263-1275.

Goldman-Rakic PS, Schwartz ML (1982) Interdigitation of contralateral 
and ipsilateral columnar projections to frontal association cortex in primates. Science 216:755-757.

Gross CG (1994) How inferior temporal cortex became a visual area. Cereb Cortex 5:455-469.

Hasegawa T, Tanaka K (1998) Neuronal activity of monkey inferotemporal cortex during tacto-visual matching task. Soc Neurosci Abstr 24:1507.

Horel JA (1994a) Local and global perception examined by reversible suppression of temporal cortex with cold. Behav Brain Res 65:157-164.

Horel JA (1994b) Retrieval of color and form during suppression of temporal cortex with cold. Behav Brain Res 65:165-172.

Horel JA, Pytko-Joiner DE, Voytko ML, Salsbury K (1987) The performance of visual tasks while segments of the inferotemporal cortex are suppressed by cold. Behav Brain Res 23:29-42.

Iwai E, Yukie M (1988) A direct projection from hippocampal field CA1 to ventral area TE of inferotemporal cortex in the monkey. Brain Res 444:397-401.

Iwai E, Aihara T, Hikosaka K (1987) Inferotemporal neurons of the monkey responsive to auditory signal. Brain Res 410:121-124.

Janssen P, Vogels R, Joris PX, Orban GA (1999) Inferior temporal neurons are selective for small differences in 3D structure. Soc Neurosci Abstr 25:529.

Jones EG, Dell'anna ME, Molinari M, Rausell E, Hashikawa T (1995) Subdivisions of macaque monkey auditory cortex revealed by calciumbinding protein immunoreactivity. J Comp Neurol 362:153-170.

Kalivas PS, Nakamura M (1999) Neural systems for behavioral activation and reward. Curr Opin Neurobiol 9:223-227.

Martin-Elkins CL, Horel JA (1992) Cortical afferents to behaviorally defined regions of the inferior temporal and parahippocampal gyri as demonstrated by WGA-HRP. J Comp Neurol 321:177-192.

Maunsell JHR, Van Essen DC (1983) The connections of the middle temporal visual area (MT) and their relationship to a cortical hierarchy in the macaque monkeys. J Neurosci 3:2563-2586.

Mistlin AJ, Perrett DI (1990) Visual and somatosensory processing in the macaque temporal cortex: the role of expectation. Exp Brain Res $82: 437-450$.

Morel A, Bullier J (1990) Anatomical segregation of two cortical visual pathway in the macaque monkey. Vis Neurosci 4:555-578.

Oram MW, Perrett DI (1994) Responses of anterior superior temporal polysensory (STPa) neurons to biological motion stimuli. J Cognit Neurosci 6:99-116.

Oram MW, Perrett DI (1996) Integration of form and motion in the anterior superior temporal polysensory area (STPa) of the macaque monkey. J Neurophysiol 76:109-129.

Perrett DI, Simth PAJ, Mistlin AJ, Chitty AJ, Head AS, Potter DD, Broennimann R, Milner AD, Jeeves MA (1985) Visual analysis of body movements by neurons in the temporal cortex of the macaque monkey: a preliminary report. Behav Brain Res 16:153-170.

Perrett DI, Harries MH, Bevan R, Thomas S, Benson PJ, Mistlin AJ, Chitty AJ, Hietanen J, Ortega JE (1989) Frameworks of analysis for the neural representation of animate objects and actions. J Exp Biol 146:87-114.

Rockland KS, Pandya DN (1979) Laminar origins and terminations of cortical connections of the occipital lobe in the rhesus monkey. Brain Res 179:3-20.

Saint-Cyr JA, Ungerleider LG, Desimone R (1990) Organization of visual cortical inputs to the striatum and subsequent outputs to the pallido-nigral complex in the monkey. J Comp Neurol 298:129-156.

Saleem KS, Hashikawa T (1998) Connections of anterior inferotemporal area TE and perirhinal cortex with the hippocampal formation in the macaque monkey. Soc Neurosci Abstr 24:898.

Saleem KS, Hashikawa T (1999) Immunohistochemical parcellation of the inferotemporal cortex and medial temporal lobe structures in macaque monkeys. Soc Neurosci Abstr 25:917.

Saleem KS, Tanaka K (1996) Divergent projections from the anterior inferotemporal area TE to the perirhinal and entorhinal cortices in the macaque monkey. J Neurosci 16:4757-4775.

Saleem KS, Tanaka K, Rockland KS (1993) Specific and columnar projection from area TEO to TE in the macaque inferotemporal cortex. Cereb Cortex 3:454-464.
Saleem KS, Cheng K, Suzuki W, Tanaka K (1995) Differential cortical projection of dorsal and ventral sub-regions of the area TE in the macaque inferotemporal cortex. IBRO Abstr 4:284.

Saleem KS, Suzuki W, Tanaka K (1996) Differential cortical connections of the dorsal and ventral sub-regions of the area TE in the macaque inferotemporal cortex. Soc Neurosci Abstr 22:1615.

Selemon LD, Goldman-Rakic PS (1988) Common cortical and subcortical targets of the dorsolateral prefrontal and posterior parietal cortices in the rhesus monkey: evidence for a distributed neural network subserving spatially guided behavior. J Neurosci 8:4049-4068.

Seltzer B, Pandya DN (1978) Afferent cortical connections and architectonics of the superior temporal sulcus and surrounding cortex in the rhesus monkey. Brain Res 149:1-24.

Seltzer B, Pandya DN (1989a) Frontal lobe connections of the superior temporal sulcus in the rhesus monkey. J Comp Neurol 281:97-113.

Seltzer B, Pandya DN (1989b) Intrinsic connections and architectonics of the superior temporal sulcus in the rhesus monkey. J Comp Neurol 290:451-471.

Seltzer B, Pandya DN (1991) Post-rolandic cortical projections of the superior temporal sulcus in the rhesus monkey. J Comp Neurol 312:625-640.

Seltzer B, Pandya DN (1994) Parietal, temporal, and occipital projections to cortex of the superior temporal sulcus in the rhesus monkey: a retrograde tracer study. J Comp Neurol 343:445-463.

Seltzer B, Cola MG, Gutierrez C, Massee M, Weldon C, Cusick CG (1996) Overlapping and nonoverlapping cortical projections to cortex of the superior temporal sulcus in the rhesus monkey: double anterograde tracer studies. J Comp Neurol 370:173-190.

Shiwa T (1987) Corticocortical projections to the monkey temporal lobe with particular reference to the visual processing pathways. Arch Ital Biol 125:139-154.

Suzuki WA, Amaral DG (1994) Perirhinal and parahippocampal cortices of the macaque monkey: cortical afferents. J Comp Neurol 350:497-533.

Tanaka K (1993) Neuronal mechanisms of object recognition. Science 262:685-688.

Tanaka K (1997) Columnar organization in the inferotemporal cortex. In: Cerebral cortex: extrastriate cortex in primates, Vol 12 (Rockland KS, Kaas JH, Peters A, eds), pp 469-498. New York: Plenum.

Turner BH, Mishkin M, Knapp M (1980) Organization of the amygdalopetal projections from modality-specific cortical association areas in the monkey. J Comp Neurol 191:515-543.

Van Essen DC, Maunsell JHR, Bixby JL (1981) The middle temporal visual area in the macaque: myeloarchitecture, connections, functional properties, and topographic organization. J Comp Neurol 199:293-326.

Van Hoesen GW, Pandya DN (1975) Some connections of the entorhinal (area 28) and perirhinal (area 35) cortices of the rhesus monkey. I. Temporal lobe afferents. Brain Res 95:1-24.

Webster MJ, Ungerleider LG, Bachevalier J (1991) Connections of inferior temporal areas TE and TEO with medial temporal-lobe structures in infant and adult monkeys. J Neurosci 11:1095-1116.

Webster MJ, Bachevalier J, Ungerleider LG (1994) Connections of inferior temporal areas TEO and TE with parietal and frontal cortex in macaque monkeys. Cereb Cortex 5:470-483.

Yukie M, Iwai E (1988) Direct projections from the ventral TE area of the inferotemporal cortex to hippocampal field CA1 in the monkey. Neurosci Lett 88:6-10.

Yukie M, Takeuchi H, Hasegawa Y, Iwai E (1990) Differential connectivity of inferotemporal area TE with the amygdala and the hippocampus in the monkey. In: Vision, memory, and the temporal lobe (Iwai E, Mishkin M, eds), pp 129-135. New York: Elsevier.

Yukie M, Hikosaka K, Iwai E (1992) Organization of cortical visual projections to the dorsal and ventral parts of area TE of the inferotemporal cortex in macaques. Soc Neurosci Abstr 18:294.

Zeki S (1974) Functional organization of a visual area in the posterior bank of the superior temporal sulcus of the rhesus monkey. J Physiol (Lond) 236:549-573. 